\title{
Gait metrics analysis utilizing single-point inertial measurement units: a systematic review
}

\author{
Ralph Jasper Mobbs ${ }^{1,2,3}$, Jordan Perring ${ }^{1,2}$, Suresh Mahendra Raj ${ }^{1}$, Monish Maharaj ${ }^{1,2}$, Nicole Kah Mun Yoong ${ }^{1,2}$, \\ Luke Wicent $\mathrm{Sy}^{4}$, Rannulu Dineth Fonseka ${ }^{1,2} \wedge$, Pragadesh Natarajan ${ }^{1,2}$, Wen Jie Choy ${ }^{1,2}$ \\ ${ }^{1}$ Faculty of Medicine, University of New South Wales, Sydney, Australia; ${ }^{2}$ NeuroSpine Surgery Research Group (NSURG), Sydney, Australia; \\ ${ }^{3}$ Department of Neurosurgery, Prince of Wales Hospital, Sydney, Australia; ${ }^{4}$ Graduate School of Biomedical Engineering, University of New South \\ Wales, Sydney, Australia \\ Contributions: (I) Conception and design: All authors; (II) Administrative support: RJ Mobbs, J Perring; (III) Provision of study materials or patients: \\ None; (IV) Collection and assembly of data: All authors; (V) Data analysis and interpretation: All authors; (VI) Manuscript writing: All authors; (VII) \\ Final approval of manuscript: All authors. \\ Correspondence to: Dr. Ralph Jasper Mobbs. Faculty of Medicine, University of New South Wales, Sydney, Australia. Email: r.mobbs@unsw.edu.au.
}

Background: Wearable sensors, particularly accelerometers alone or combined with gyroscopes and magnetometers in an inertial measurement unit (IMU), are a logical alternative for gait analysis. While issues with intrusive and complex sensor placement limit practicality of multi-point IMU systems, single-point IMUs could potentially maximize patient compliance and allow inconspicuous monitoring in daily-living. Therefore, this review aimed to examine the validity of single-point IMUs for gait metrics analysis and identify studies employing them for clinical applications.

Methods: The Preferred Reporting Items for Systematic Reviews and Meta-Analyses Guidelines (PRISMA) were followed utilizing the following databases: PubMed; MEDLINE; EMBASE and Cochrane. Four databases were systematically searched to obtain relevant journal articles focusing on the measurement of gait metrics using single-point IMU sensors.

Results: A total of 90 articles were selected for inclusion. Critical analysis of studies was conducted, and data collected included: sensor type(s); sensor placement; study aim(s); study conclusion(s); gait metrics and methods; and clinical application. Validation research primarily focuses on lower trunk sensors in healthy cohorts. Clinical applications focus on diagnosis and severity assessment, rehabilitation and intervention efficacy and delineating pathological subjects from healthy controls.

Discussion: This review has demonstrated the validity of single-point IMUs for gait metrics analysis and their ability to assist in clinical scenarios. Further validation for continuous monitoring in daily living scenarios and performance in pathological cohorts is required before commercial and clinical uptake can be expected.

Keywords: Accelerometry; gait analysis; wearable electronic devices

Received: 12 April 2021; Accepted: 27 August 2021; Published: 20 January 2022.

doi: $10.21037 /$ mhealth-21-17

View this article at: https://dx.doi.org/10.21037/mhealth-21-17

\section{Introduction}

Human gait is affected by ageing as well as numerous musculoskeletal and neurological ailments. Consequently, gait analysis has wide-ranging clinical applications from diagnosis and severity assessment as well as evaluation of intervention and rehabilitation efficacy in neurological and

\footnotetext{
$\wedge$ ORCID: 0000-0002-7748-5101.
} 
orthopedic conditions (1-8) to identifying falls risk and frailty status $(9,10)$. Qualitative and subjective measures generally constitute routine clinical gait analysis, with patient self-reporting and clinician observation sometimes integrated with clinical tests such as the Timed-Up-And-Go and 6-minute walking test (6MWT) (11). These approaches impose significant interobserver inaccuracies and deny appreciation of kinematic and kinetic intricacies that can be obtained from quantitative gait assessment (12).

The gold-standard for quantitative gait analysis, optoelectronic stereophotogrammetry, features infrared cameras that capture three-dimensional trajectories of reflective markers placed on the subject that are processed to accurately assess spatio-temporal and kinematic variables of gait (13). Stereophotogrammetry is often combined with force plates that measure ground-reaction forces (GRF) to determine kinetic forces and electromyography (EMG) systems to measure muscle activity during gait. However, these systems are expensive, time-consuming, and require expert operation and equipment. Furthermore, restriction of their performance to dedicated laboratory settings limits portability, access and external validity of measures obtained to free-living gait (11).

In response, wearable sensors (goniometers, EMG systems, sensing fabric etc.), particularly accelerometers alone or combined with gyroscopes and magnetometers in an inertial measurement unit (IMU), are proving to be the logical alternative for gait analysis. Cheap, small and portable, wearables could potentially enable continuous gait metrics analysis in daily living (14-17). Furthermore, fast preparation and processing negate the need for expert operation, enhancing practicality. Multi-point IMU systems have been validated against standards (18-23) and employed clinically for gait metrics analysis (24-28); however, issues with intrusiveness and consistency of complex sensor placement limit real-life adoption (29). Although not as accurate and reliable as multi-point IMU systems (30), single-point IMUs have nonetheless demonstrated enormous potential. They have clinical uses, such as with the assessment of Parkinson's Disease (PD) severity $(31,32)$ and the evaluation of falls risk in preventative health care $(33,34)$, and personal uses, such as with the tallying of daily steps in consumer-grade watches $(4,35)$. Commonly used single-point IMUs and their specifications are detailed for comparison in Table 1.

Moreover, presuming validated accuracy is demonstrated, single-point IMUs could potentially maximize patient compliance and comfort and allow inconspicuous monitoring in daily-living. Consequently, any future mass uptake of IMUs in clinical and commercial settings is likely to be dependent upon the validation and applications of single-point IMUs and not of multi-point IMU systems. Therefore, this review will provide a synopsis of inertial sensor principles, practical considerations and gait metrics analysis capabilities before examining the validity and clinical applications of single-point devices for gait analysis. We present the article in accordance with the Preferred Reporting Items for Systematic Reviews and Meta-Analyses Guidelines (PRISMA) checklist (available at https://dx.doi.org/10.21037/mhealth-21-17).

\section{Inertial sensors: principles and practical considerations}

Inertial sensors, accelerometers and gyroscopes, are often fabricated into microelectromechanical systems (MEMS) alone or together as an IMU and employed for gait metrics analysis (78).

Accelerometers measure acceleration along their sensitive axis, ranging from uni-axial to the commonly employed tri-axial sensitivity which allows appreciation of movement along the antero-posterior, horizontal and vertical planes. However, current devices are susceptible to drift errors due to change in mechanical or electrical properties and noise from amplified mechanical motions. Furthermore, the measured acceleration comprises both the inertial acceleration associated with changes in velocity, and gravitational acceleration superimposed along the accelerometer's sensitive axes. Removing this confounding effect of gravity can be difficult (78). To appreciate velocity and distance, numerical integration of acceleration data is required, causing noise and drift errors to accumulate, imposing significant limitations on long-term accelerometer employment. Without compensation for this drift, readings become useless. Compensation requires frequent accelerometer recalibration, achieved through zero-velocity updates (ZUPT), using an external event indicating an instantaneous null in movement such as a footstep (79).

Gyroscopes measure angular velocity, demonstrating greater accuracy than accelerometers as measurement is absolute with no external information considered (80). However, gyroscopes only return rate of change of angular position; to detect relative orientation, integration of the signal is required. This leads to accumulation of drift errors and noise, similar to accelerometers (80). Furthermore, lack of an initial reference compared to accelerometers means gyroscopes cannot be recalibrated, resulting in accumulation of errors and limited long-term 


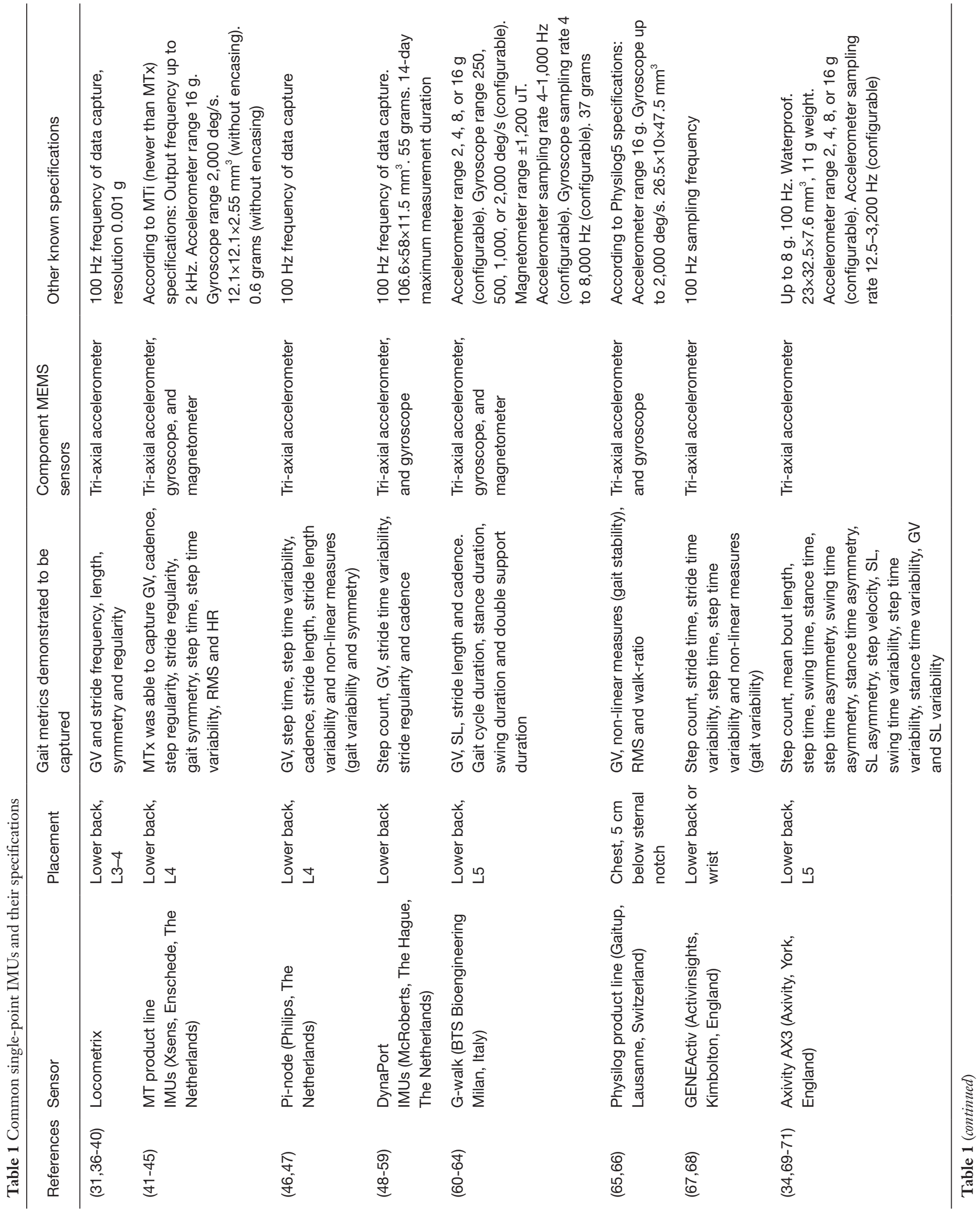




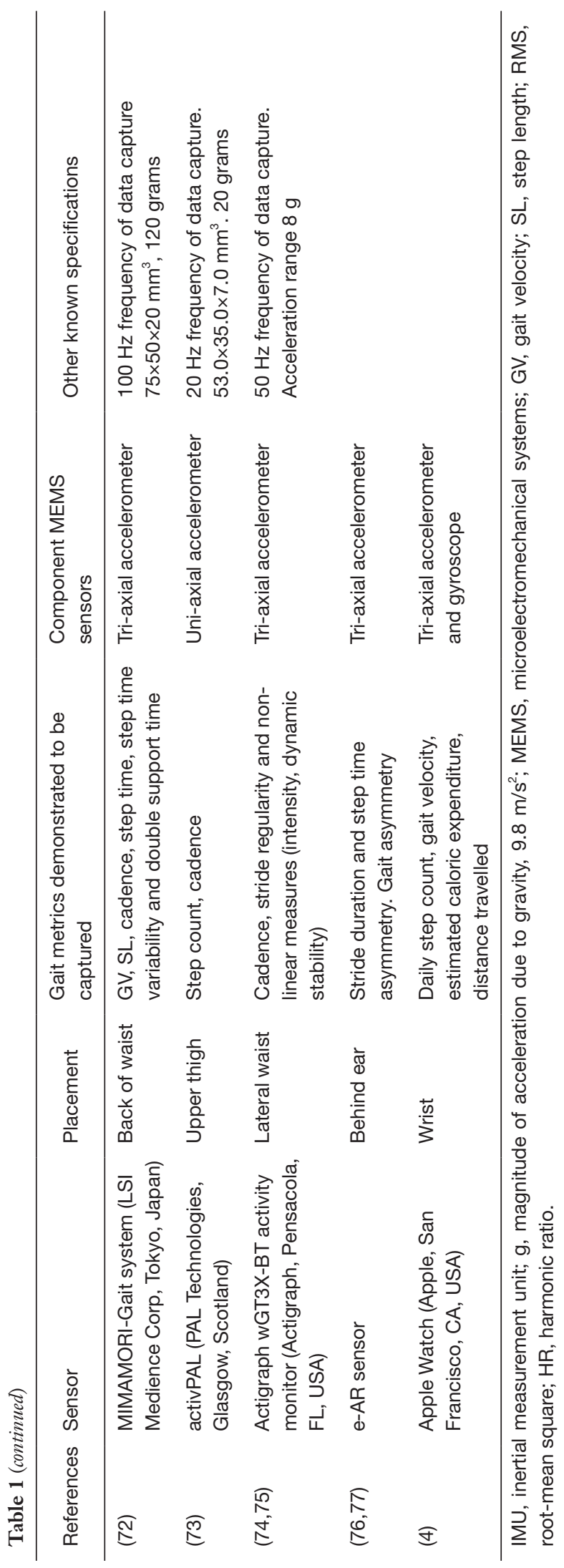

precision (80). This limitation is often minimized by incorporating a magnetometer in the IMU, able to calibrate sensor orientation with reference to the Earth's magnetic field. However, these devices are prone to interference by magnetic fields created by other devices (80).

\section{Inertial sensor-based gait analysis}

While single-point inertial sensors are unable to appreciate kinetic and many kinematic variables of gait, they can determine spatio-temporal parameters. Spatiotemporal parameters are of importance clinically, as they objectively characterize key gait events (GE) and common gait abnormalities (81). A plethora of spatio-temporal parameters are employed in the literature, with some [such as gait velocity (GV) and gait regularity in predicting the staging of PD severity] being more relevant than others in different clinical scenarios $(31,32)$. This review focusses on spatio-temporal parameters based on a validated model $(82,83)$ and clinical guidelines from The Biomathics and Canadian Gait Consortiums Initiative (84). These parameters encompass the mean, variability and asymmetry of temporal (cadence, step time, stride time, stance duration, swing duration, single-support duration, double-support duration) and spatial [step length (SL), stride length, GV step width] components of gait.

As acceleration data retains a time-series nature when extracted, by determining GEs such as heel-strike (HS) and toe-off (TO) within the gait cycle, mean temporal parameters can be quantified. Methods for GE detection are based on signal feature extraction of peaks, valleys or zerocrossings from raw accelerometric or gyroscopic data that may indicate a HS or TO (78). This can be complemented by applying hidden Markov models or Gaussian continuous wavelet-transformation (CWT) to increase GE detection accuracy (29). These methods have been implemented for single sensors placed on the trunk $(85-87)$, waist $(88,89)$, shank (90), ear (76) and foot $(91,92)$. A thigh-based singlepoint IMU, the Activpal (PAL Technologies, Glasgow, Scotland), has also been used to measure mobility $(73,93)$. However, this sensor has, to our knowledge, not analysed gait metrics beyond step identification in a single-point system.

Spatial parameter estimation proves more difficult due to the aforementioned technical limitations of inertial sensors (29). Current methods are based on abstraction models (e.g., machine-learning, linear regressive models), locomotion models [e.g., inverted pendulum (IP), double- 
IP] and numerical integration. Those employed for singlepoint sensors include: direction integration (88), linear regression models (89), IP model with double-integration of antero-posterior $(86,87)$ or vertical acceleration from trunk sensors (85); IP model with double integration of AP-acceleration from a foot sensor (91); double-IP model with integration of angular velocity of the shank (90); and autocorrelation procedures also able to determine temporal parameters, and a measure of regularity and symmetry (94). However, the requirement of numerical integration in these models causes accumulation of drift errors (78). Drift compensation is performed using kinematical reset through ZUPT by assuming foot velocity as zero (91) and shank inclination as vertical during midstance (90); however, these methods only prevent growth of drift error without minimizing the already accumulated error (29). As zero-velocity reset is not possible with trunk sensors due to continuous pelvic motion, drift correction is achieved by applying a highpass (86) or Kalman filter (88) to retrospectively correct errors, occasionally in combination with direct and inverse integration at every step (88). However, correction efficacy may be limited in pathological gait where vertical trunk acceleration amplitude is lower and variability higher (78).

Linear measures of spatio-temporal variability and asymmetry are subsequently determined from mean spatio-temporal values, commonly expressed as the standard deviation or coefficient of variation and the absolute difference between left and right mean values respectively (95).

In addition to these traditional spatio-temporal parameters, accelerometer-based systems and non-linear calculations introduce new measures (11). Although these complex non-linear, autocorrelation and acceleration-based measures are dimensionless and unable to be validated against a standard, they are employed extensively clinically. Non-linear measures derived from the theory of stochastic dynamics (e.g., phase plot analysis, fractal-scaling index, sample entropy, Lyapunov exponents) allow appreciation of dynamic fluctuations and patterns between gait cycles throughout a walking bout, contrary to traditional linear measures that treat each as independent to the last $(11,49,83)$. These measures represent the smoothness, regularity, stability, variability, complexity and symmetry of gait, showing sensitivity delineating between pathological and healthy subjects (96-98) equal, or superior to the sensitivity of linear measures (99). Similarly, autocorrelation measures of regularity and symmetry represent a clinically relevant $(31,44,67)$, dynamic substitute for spatio-temporal variability and symmetry respectively $(36,94)$. Other measures, including harmonic ratio (HR) based on Fourier analysis and the root-mean square (RMS) of acceleration magnitudes, are clinically relevant indicators of the smoothness, rhythmicity and symmetry of gait $(33,100-102)$.

\section{Methods}

\section{Literature Search}

The PRISMA guidelines were followed for this systematic review (103) utilizing the following databases: PubMed; MEDLINE; EMBASE; Cochrane. Firstly, key search terms "gait" AND "accelerometer or inertial" were used to locate studies using inertial sensors to monitor gait. Next, "spatiotemporal" or "temporal" or "phase" or "stride" or "length" or "velocity" were used to locate publications that measure clinically relevant gait metrics beyond stepcount and activity. Finally, the terms "clinical or valid or validity or test or reference or standard" were included to reflect studies that had tested the validity of these wearable technologies or applied them clinically. Relevant MeSH (Medical Subject Heading) terms, variations and synonyms were adjusted for each database.

\section{Study selection}

Studies from the above databases were collated and duplicate studies removed. Primary screening by an independent reviewer (JP) was performed based on the title and abstract of the remaining studies following the developed inclusion and exclusion criteria detailed below. Subsequent eligibility assessment was performed based on the full texts of remaining articles by an independent reviewer (JP) following the inclusion and exclusion criteria.

\section{Inclusion criteria}

(I) Articles involving wearable technology/ies.

(II) The wearable technology features an inertial sensor (accelerometer, gyroscope) or is an IMU.

(III) The wearable technology is a single-point sensor.

(IV) Articles written in English.

(V) Journal papers. 


\section{Exclusion criteria}

(I) Wearable technology/ies only capable of identifying activity or step count.

(II) Wearable technology/ies features multiple sensor points.

(III) Wearable technology/ies classed as robotic or exoskeletons.

(IV) Systematic reviews, books, or conference papers.

\section{Data collection}

Following final article selection, results were classified as validation, clinical application or both. Data for validation studies was collected including sensor type(s); sensor placement; study aim(s); conclusions of study; primary gait metrics and methods. Critical analysis of validation studies was also included. Data collected for clinical applications studies included: sensor type(s); sensor placement; primary gait metrics and methods; and clinical application.

\section{Bias analysis}

Three different tools were used for risk of bias assessment based on the nature of the studies. Validation and reliability studies were assessed using the Scottish Intercollegiate Guidelines Network (SIGN) methodology checklist for diagnostic tests $(104,105)$. Of the clinical application studies, observational studies were assessed using the Newcastle-Ottawa Scale (106) while randomized-controlled trials (RCTs) were assessed using the SIGN checklist for RCTs $(105,107)$. Case series and case reports were excluded from the use of these bias assessment scales as questions pertaining to comparability no longer apply. Studies were assessed by 3 independent reviewers (WJC), (SMR) and $(\mathrm{MM})$ with at least 2 different reviewers for each study. Discrepancies in assessment were resolved by discussion and reaching a consensus.

\section{Results}

From the 1,068 articles retrieved after duplicate removal, 90 articles were selected for inclusion (Figure 1). Thirtytwo articles assessed the validity of single-point sensors and 48 used single-point sensor gait metrics analysis for clinical applications. Ten articles concurrently assessed validity and employed the device in a clinical application.

\section{Validity of single-point sensors}

Among the 42 articles (Figure 2), 30 studies utilized trunkbased sensor methods; while 12 studies used alternate locations for the sensor placement which include four studies at the subjects' ear; two at the subjects' shank, two at the subjects' foot, one at the waist; while three other studies utilized smart devices with inertial sensors. The parameters used for validating the sensors include: HS, stride length/duration/regularity, SL/count/duration/ length variability/variability/time asymmetry/cycle time/ regularity/frequency, GV, cadence, traversed distance, walking time, stance duration, swing duration, single/ double support duration, HR, TO and time averaged acceleration.

\section{Validation studies by subject cohort}

Among the 42 studies, 59 different cohort of patient population were studied (Figure 3). Twenty-five studies involved healthy adults, 11 studies involved elderly subjects, seven studies involved patients with $\mathrm{PD}$, four studies in post-stroke patients with ataxia and three studies in Huntington's Disease (HD). There were two studies each for patients with lower limb amputees and diabetic patients. The remainders were single studies in subjects comprising healthy children, multiple sclerosis (MS) patients, orthopedic patients, muscular dystrophy patients, and patients suffering from motor neuron disease (Table S1).

\section{Clinical application of single-point IMUs}

Fifty-eight articles discussed the use of single-point IMUs in clinical setting. Of these, 12 articles discussed the application of sensors in diagnosis and assessing severity of diseases (PD, MS, PN, Alzheimer's disease, age-related changes, frailty and foot \& ankle health); 12 studies applied the sensors in monitoring rehabilitation and intervention efficacy (orthopedic, neurosurgical and oncological patients, foot orthoses, medical and physical intervention in neurodegenerative diseases); and 31 studies used sensors to characterize patients with different conditions from healthy subjects. Three articles described both diagnosis and severity of assessment as well as delineating healthy and participants with pathologies (Table 2, with more detailed findings of these articles in Tables S1,S2. 


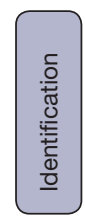

Database search $(n=1,851)$

PubMed $(n=505)$

MEDLINE $(n=379)$

EMBASE $(n=902)$

Cochrane library $(n=65)$

Studies retrieved by expertcontact, handsearching of references $(n=12)$
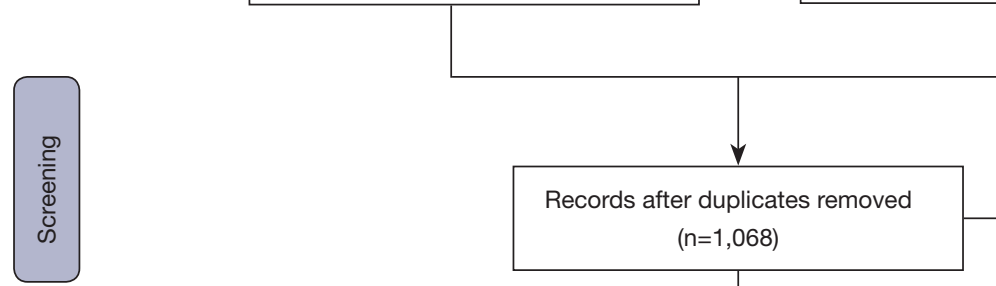

Records after duplicates removed $(n=1,068)$

Records excluded $(n=775)$
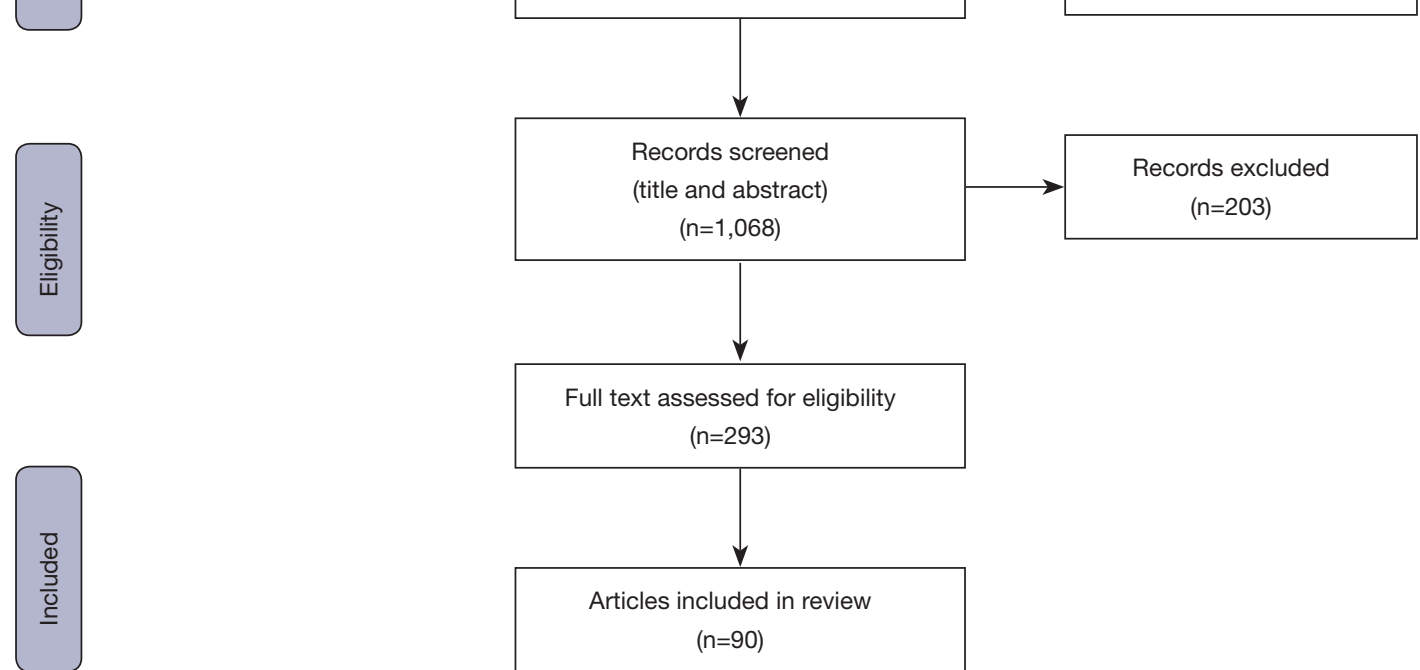

Figure 1 PRISMA methodology.

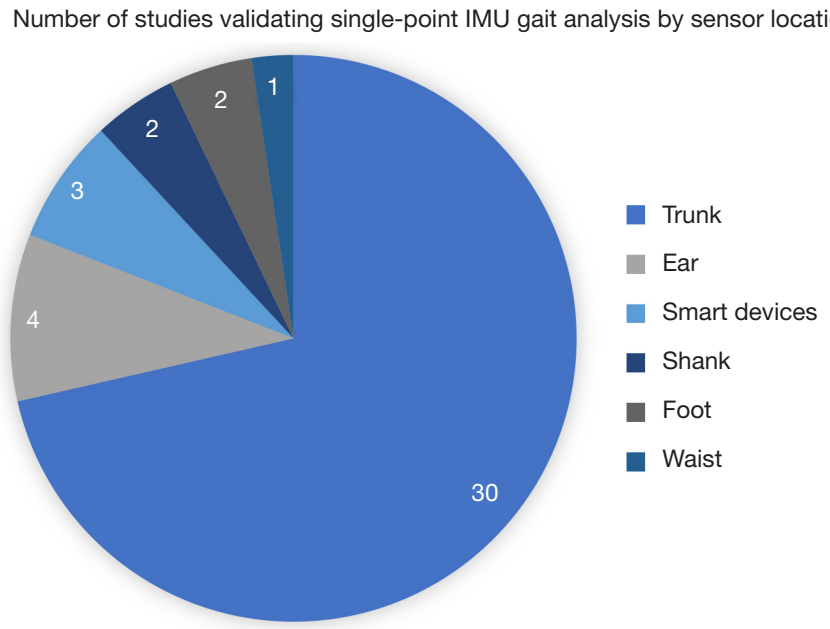

Figure 2 Number of studies validating single-point IMU gait metrics analysis by sensor location. Smart devices have been attached on various locations, some separate to where they were designed to be worn - the iPod touch G4 (iOS 6, Apple Inc.) over L3 (108), the Apple Watch (Apple, San Francisco, CA, USA) on the wrist (4), and the Apple iPhone 5 (iPhone 5, Apple Inc., Cupertino, CA, USA) on the lateral waist (109). IMU, inertial measurement unit. 


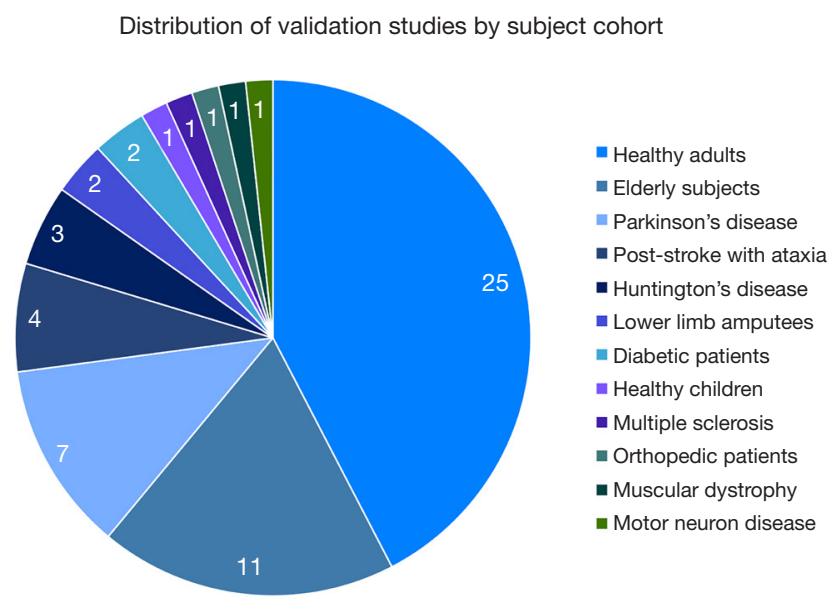

Figure 3 Distribution of validation studies by subject cohort.

\section{Bias assessment}

Risk of bias assessment of validity studies did not reveal studies with an unacceptable level of bias. 31 studies were of high quality while nine were of acceptable quality in minimizing bias. The breakdown of the assessment and interpretation is included in Table S3. Areas in which many studies had 'unclear' levels of bias were in the patient selection domain criteria of selecting a consecutive sequence or random selection of participants. Understandably, many of these studies had small sample sizes and recruited volunteers or practiced convenience sampling of patients to achieve this. Many studies were able to reduce bias by conducting simultaneous testing of the IMU and reference standard. Criteria related to the use of a reference standard were not applied to reliability studies that did not use a reference test. The criteria related to pre-specified thresholds of the index test were also largely not applicable.

Of the clinical applicability studies, 30 were scored as "good" quality, eight as "fair" and 12 as "poor". Two RCTs were deemed having acceptable quality and one RCT as high quality in minimizing bias. The breakdown of the bias assessment results and interpretation is included in Appendix 1). Most studies generally missed out a score on ascertainment of outcome using a blind investigation. This may be attributed to a lack of investigators needed to separate carrying out the test and interpreting data. However, this was regarded as not having a large influence on overall bias assessment as measurements using IMUs are automatically recorded to software and not requiring direct human measurement. It would also be difficult to blind assessors to diseased patients with an obvious gait pathology to healthy controls. The RCTs also had unclear blinding of subjects and investigators to treatment groups. The strengths of these studies were the randomization process and standardization of testing and analysis between treatment and control groups.

\section{Discussion}

In our review, single-point IMUs have been reasonably validated in the measurement of spatial and temporal gait parameters $(85,86,124)$. However, IMUs have shown difficulty in estimating variability and asymmetry metrics (48,70,118,125-127). Alongside this, whilst IMUs have shown promise in their clinical applications, such as in the diagnosis of disease $(31,32,47,53)$ and the assessment of treatment efficacy $(56,113,128)$, these studies have predominantly relied on straight-line gait metrics. This critically limits external validity to free-living analysis where day-to-day movements typically represent more complex patterns of acceleration and deceleration. Moreover, studies focusing on clinical application have predominantly described obvious gait changes and have not necessarily demonstrated IMUs to be useful in evaluating subtle differences in gait patterns. Therefore, additional studies focusing on validation and clinical application are required before any mass clinical and commercial uptake of singlepoint wearable sensors can occur. 
Table 2 Clinical applications of single-point IMUs

\begin{tabular}{|c|c|}
\hline Reference & Application \\
\hline \multicolumn{2}{|c|}{ Diagnosis and severity assessment } \\
\hline Demonceau et al., 2015 (31) & Determine PD severity \\
\hline Herman et al., 2014 (57) & Classify PD subtypes \\
\hline $\begin{array}{l}\text { Dalton et al., } 2013 \\
\text { Collett et al., } 2014 \\
\text { Pau et al., } 2016 \\
(47,62,110)\end{array}$ & Determine MS severity \\
\hline Esser et al., 2018 (111) & Detect PN \\
\hline De Bruin et al., 2012 (53) & Determine PN severity \\
\hline Gillain et al., 2016 (39) & Predict risk of Alzheimer's development \\
\hline $\begin{array}{l}\text { Soangra et al., } 2018 \\
\text { Martinez-Ramirez et al., } 2015 \\
(43,109)\end{array}$ & Predict frailty status/determine severity \\
\hline $\begin{array}{l}\text { Van Schooten et al., } 2016 \\
\text { Del Din et al., } 2017 \\
(33,34)\end{array}$ & Predict falls/determine falls risk \\
\hline Angthong et al., 2018 (112) & Assessment of foot/ankle conditions \\
\hline \multicolumn{2}{|c|}{ Rehabilitation and intervention efficacy } \\
\hline $\begin{array}{l}\text { Atallah et al., } 2014 \\
\text { Rapp et al., } 2015 \\
(76,113)\end{array}$ & Total hip and knee replacement efficacy and recovery \\
\hline $\begin{array}{l}\text { Mutoh et al., } 2016 \\
\text { Manikowska et al., } 2013 \\
(5,56)\end{array}$ & Hippotherapy efficacy in cerebral palsy \\
\hline Henderson et al., 2016 (114) & Rivastigmine efficacy in PD \\
\hline Terrier et al., 2009 (115) & Prescription footwear efficacy in foot/ankle fractures \\
\hline $\begin{array}{l}\text { Doi et al., } 2013 \\
\text { Pau et al., } 2014 \\
\text { Perrochon et al., } 2015 \\
(38,60,101)\end{array}$ & Improvement in gait after physical activity in elderly and cognitive impairment \\
\hline Delineating pathological subjec & \\
\hline $\begin{array}{l}\text { Barden et al., } 2016 \\
\text { Clermont et al., } 2016 \\
\text { Bolink et al., } 2012 \\
(67,68,116)\end{array}$ & Knee osteoarthritis subjects \\
\hline
\end{tabular}

Table 2 (continued) 
Table 2 (continued)

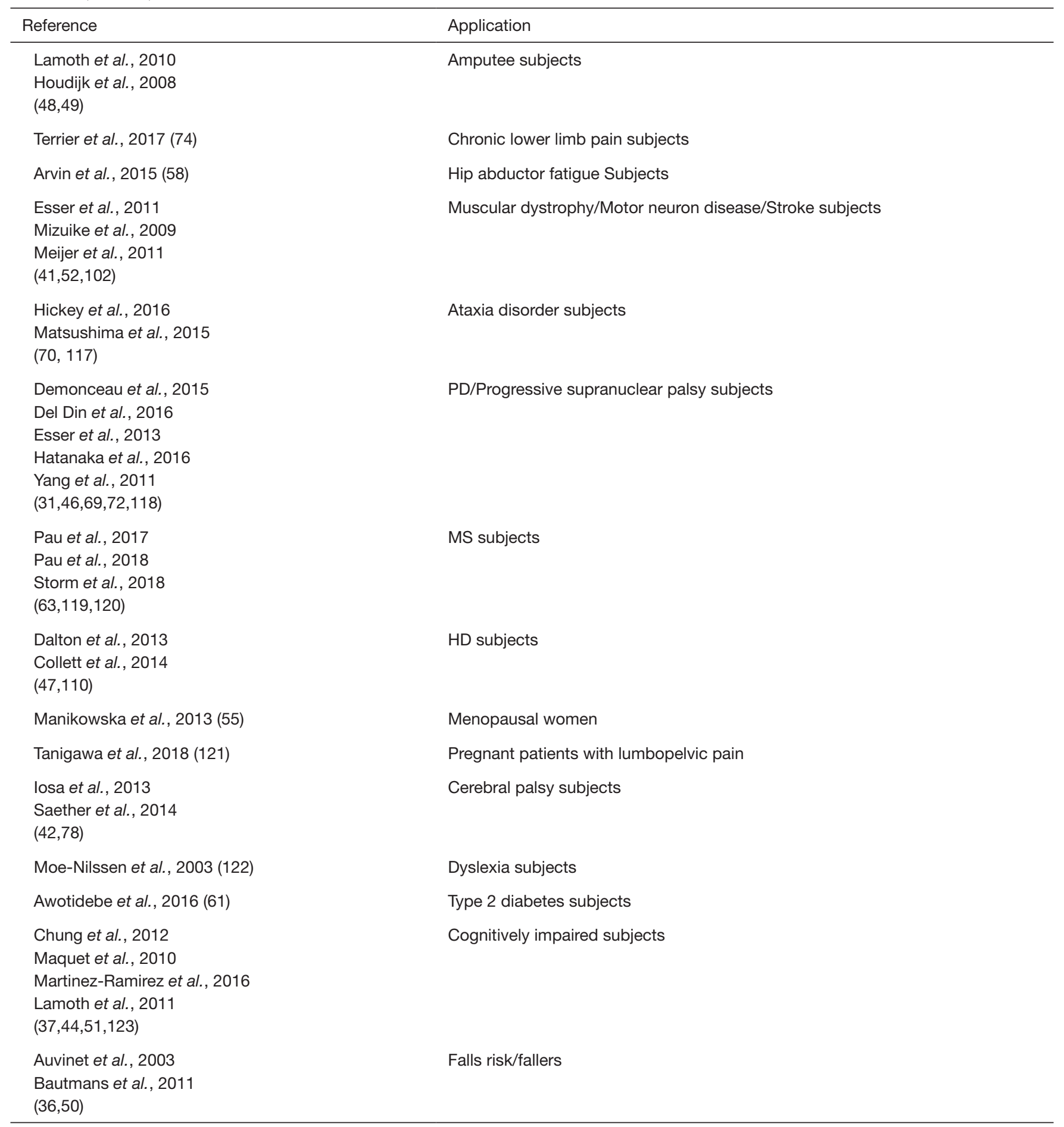

IMU, inertial measurement unit; PD, Parkinson's Disease; MS, multiple sclerosis; PN, peripheral neuropathy; HD, Huntington's Disease. Detailed findings of these articles can be found in Tables S1,S2. 


\section{Validating IMU gait metrics analysis}

Proposed methods and sensor locations for determination of gait metrics from single-point IMU acceleration data are generally validated against a standard or by test-retest reliability (129). The large portion of validation research employing lower trunk sensors compared with alternative sensor locations is reflected in Figure 2.

\section{Trunk-based sensor methods}

Association of lower trunk accelerations with HS and TO, and the ability to predict these accelerations with an IP model of the body's center of mass (COM) trajectory, has prompted the proposal of several methods for GE and spatio-temporal parameter estimation from these signals.

\section{Metbods by Zijlstra \& Hof (86)}

Peak detection and IP methods proposed by Zijlstra \& Hof (86) utilizing a tri-axial accelerometer over the lower trunk demonstrated accurate detection of GEs and limited mean spatio-temporal parameters when compared to GRF from a treadmill. However, the straight-walking protocol employed limits external validity to daily-living analysis. Similarly, $100 \%$ of GEs were detected in only nine subjects of a small sample $(n=15)$ of healthy adults, with $12 \%$ of GEs identified falsely in the remaining subjects. Furthermore, SL calculation which requires input of individual leglength was consistently underestimated before application of a 1.25 correction factor, revealing limitations in the model itself. Further validation in healthy adults has demonstrated accurate estimation of mean spatio-temporal parameters when compared to stereophotogrammetry and dynamometry, however significant differences were found for gait phase durations that rely on determining TO which isn't explicitly detected by the model (124). SL estimation was again detected with less accuracy due to errors implied by double-integration in this method (124). Further model limitations were identified in neurological populations (41) as neither a generic (1.25) or pathology-specific correction factor could be applied for accurate SL estimation, alluding to the need for individual corrections in pathological cohorts. This finding was reflected when validated in child (130) and older cohorts (131). The model has been further validated in small samples of PD subjects against motion capture (132), MS subjects against an instrumented walkway (110) and through test-retest reliability (47) for mean and variability spatio-temporal measures. These studies also assessed reliability of non-linear variability measures (47) and demonstrated feasibility of anterior trunk sensor placement, representing an attenuated version of COM accelerations (110). Methods from Zijlstra and Hof (86) have been incorporated into commercially available IMUs, G-Walk (BTS, Milan, Italy) and DynaPort (McRoberts, The Hague, Netherlands). DynaPort accuracy has been assessed in children (130), lower-limb prosthesis (48), diabetes $(53)$, healthy elderly $(125,126)$ and falls risk subjects (50), while G-walk accuracy has been assessed in healthy adult (133-135), PD (136) and MS (137) subjects. Despite determining mean spatiotemporal parameters accurately in most studies, caution is recommended for interpretation of linear variability and asymmetry parameters $(48,125,126)$. The interpretation of gait phase durations reliant on TO (stance, swing, single, double support time) is also uncertain since this event is not explicitly detected by the algorithm $(133,136,137)$. Furthermore, testing limited to small samples in controlled straight-walking conditions limits power of inference to gait metrics analysis in pathological cohorts or scenarios of daily-living.

Autocorrelation methods utilizing lower trunk accelerations from a tri-axial accelerometer (94) have demonstrated test-retest reliability in a small sample $(n=20)$ of healthy subjects for RMS and mean spatiotemporal parameter assessment (138) in straight-walking protocols. Despite lacking further validation for traditional spatio-temporal parameters, the test-retest reliability of autocorrelation measures of regularity and symmetry have been demonstrated in small samples of elderly, falls-risk and HD subjects in straight-walking protocols $(47,50,139)$.

\section{Methods by McCamley et al. (85)}

CWT for GE detection and IP methods for spatial parameter estimation proposed by McCamley et al. (85) using a lower trunk IMU have been validated against stereophotogrammetry and force plates. It has also been compared against previous methods from Zijlstra \& Hof (86) and González et al. (87). Despite limitations of a small sample $(\mathrm{n}=18)$ of healthy subjects and a straightwalking protocol of only $3.6 \mathrm{~m}$, the method by McCamley et al. (85) provided an improved estimate of SL and GE detection compared to previous methods. Validity for temporal parameter means and variability has been subsequently demonstrated in both controlled and freeliving conditions against pressure insoles, despite observed accuracy decreases in free-living conditions (140). Similar protocols in MS patients validated measurement of mean temporal parameters (120). However, variability measures were highly overestimated, with inaccuracies increasing 
with length of walking bout, detracting from applicability to continuous monitoring. The lower accuracy for which this method detects TO compared to HS, may account for difficulty estimating variability measures, shown to be highly sensitive to incorrect GE identification compared to mean parameters (120). Method accuracy was also shown to be speed-dependent and decrease with increasing disability, hindering reliability in pathological cohorts (120).

Method by Godfrey et al. $(95,141)$ combining those by Zijlstra \& Hof (86) and McCamley et al. (85)

Combining methods by McCamley et al. (85) for temporal parameter and Zijlstra \& Hof (86) for SL estimation, Godfrey et al. $(95,141)$ demonstrated validity against an instrumented walkway and through test-retest reliability in large populations of young and older adults (cumulative $\mathrm{n}=92$ ) in protocols reflecting daily-living. Despite acceptable agreement for mean spatio-temporal parameters, SL underestimation was again attributed to limitations with generic correction factors, straight-walking dependent IP model and mathematical integration errors. Both methods' measures of spatio-temporal variability and asymmetry were poor, concurring with findings of other studies using an instrumented walkway as a control (70,125-127). In defense of IMUs, Godfrey et al. (95) demonstrated that discrepancies in variability and asymmetry were due to inherent differences between IMUs and instrumented walkways used in these studies, rather than IMU inaccuracy. This highlights the importance of caution when choosing a standard for validation purposes. Demonstrated discriminatory power between pathological and healthy cohorts based on IMU asymmetry and variability measures, despite poor agreement with an instrumented walkway, reinforces these conclusions (70).

\section{Other methods of GE and spatio-temporal parameter estimation}

Waist-placed sensor and algorithm development has also been validated. Direct integration methods for SL estimation based on a waist placed IMU (88) were validated against stereophotogrammetry. However, limited gait parameters, a small sample $(\mathrm{n}=9)$ of healthy patients and accuracy discrepancies between left and right steps due to anatomically asymmetrical sensor placement limit clinical applicability (88).

To combat the plethora of COM methods, a comparison of five methods $(85,86,88,89,142)$ against stereophotogrammetry and force plates for determination of temporal parameters was conducted by Trojaniello et al. (143). The different GE identification methods incorporated largely either the zero-crossing or waveletbased method. Zjilstra \& Hof (86) used a zero-crossing method where foot contact was taken as peak forward acceleration preceding the change of sign of acceleration from positive to negative. González et al. also used a zerocrossing method to approximate a search window prior to applying certain heuristic rules to determine the peak associated with the contact event (142). In conjunction with the zero-crossing method, Shin $e$ al. used a sliding window summing technique to reduce noise (89). The method by McCamley et al. involved integrating and differentiating the acceleration signal using Gaussian continuous wavelet transforms prior to identifying initial and final contact events from the minima and maxima of the smoothed signal (85). Köse et al. used a wavelet-based method to identify windows of interest prior to decomposition and reconstruction of the original signal based on certain threshold application. Heel strike was then detected as the timepoint between signals of the different local frame axes (88). No statistically significant difference was found between methods for stride and step duration and the standard. However, methods that detect TO in addition to HS to allow determination of gait phase durations $(85,87,88)$ showed a statistically significant difference, due to difficulties detecting the smoother acceleration signals indicating TO (143). Omitting assessment of spatial parameter methods limits completeness of the study. While despite examining a large sample, comparison of methods in healthy controls limits external validity to pathological cohorts. In response, assessment of the three best-performing methods $(85,86,89)$ in 10 hemiparetic, 10 PD and 10 HD subjects against an instrumented walkway was undertaken (144). This revealed a universal decrease in GE detection and temporal parameter accuracy compared to healthy subjects. However, no statistically significant differences were revealed regarding accuracy between IMU methods in any cohort, apart from PD subjects for which methods from Zijlstra \& Hof (86) outperformed.

New methods are continuously being formulated for lower trunk IMU analysis of spatial and temporal parameters. Oyake et al. (145) recently proposed a new algorithm for SL symmetry determination, validated in stroke subjects, while Sejdić et al. (146) validated novel methods against motion capture data in PD and peripheral neuropathy $(\mathrm{PN})$ subjects.

\section{Alternative sensor placements}

Despite trunk IMUs maintaining the lion's-share of 
research, methods based on alternative sensor positions have also garnered validation attention.

An ear-worn tri-axial accelerometer has been validated in healthy and lower-limb orthopedic subjects against an instrumented treadmill for estimation of mean step time and symmetry (76). A similar sensor has also been validated for the detection of GE's and limited gait parameters in small samples of healthy (147), PD (148) and orthopedic (77) subjects in laboratory conditions.

With placement closer to the ground allowing better GE detection, sensors on the lower limb have also been proposed and validated. GV estimation from a single shank IMU was validated on a treadmill across numerous speeds and slope gradients (90). However, decrease in effectiveness of vertical shank inclination as a ZUPT re-calibration and subsequent GV accuracy with changing incline limits dailyliving application. Maqbool et al. (149) further validated an algorithm for detection of GEs across walking speeds and slopes in eight healthy and two amputee subjects using a shank gyroscope. Straight-walking protocols and need for the instrumented shank to take the first step limit these studies' clinical applicability. IP methods using one foot IMU have been validated against a treadmill for GV estimation in five healthy subjects (91). However, difficult attachment of the IMU to the shoe, straight-walking protocols and an accuracy decrease with increasing incline limit applicability. Temporal parameter detection has also been validated using a foot IMU in eight healthy adults (92), showing strong correlation with motion capture. Limited validation of comprehensive gait metrics and inability to assess asymmetry and complex trunk-accelerometer measures with lower-limb sensor placement limits clinical applicability (88).

\section{Smart device gait analysis}

Smart devices embedded with inertial sensors have become ubiquitous in everyday life, making them an obvious solution for maximizing patient compliance and allowing inconspicuous, portable gait analysis.

Initial proof of concept using an iPhone attached to the lateral malleolus demonstrated test-retest reliability for quantification of time averaged acceleration and step duration (150). However, unrealistic device placement, limited gait parameters and a sample of only one healthy patient limit validity and clinical applicability. Following this, utilizing trunk-based methods for GE detection (85-87) and SL estimation (86), high correlations were found for an iPhone against stereophotogrammetry for the identification of GE and mean spatio-temporal parameters in eleven healthy subjects (151). However, similar unrealistic device placement over the lumbar spine and waist has limited applicability to daily living. Addressing this, the reliability of smartphone locations: body, belt, bag, pocket and hand and validity against an instrumented walkway has been tested (152). Hand positions demonstrated poor reliability and agreement with the standard at slow speeds which only marginally improved at higher velocity, while high validity and excellent reliability were demonstrated in body, bag or belt positions at fast/comfortable speeds, lending traction to their incorporation into everyday gait monitoring. However, universal inaccuracies assessing gait at slow speeds limits application to pathological groups. Furthermore, limited emulation of free-living scenarios was employed with only five to nine steps investigated per trial (152).

\section{Validation status of single-point IMUs}

Despite lacking validation for a comprehensive set of gait metrics in alternative positions, current COM systems are a proven alternative for calculation of a range of traditional spatio-temporal measures. However, these algorithms still need development, with caution recommended with interpretation of spatio-temporal variability and symmetry parameters, measures of gait phases and estimation of spatial parameters. Further validation is required in larger samples of pathological groups; Furthermore, their accuracy for continuous monitoring in scenarios of daily living needs to be assessed. Incorporation of these algorithms into commercial devices and smart devices is promising for clinical practicality and uptake.

\section{Clinical applications}

Although the vision of single-point IMUs for gait metrics analysis in daily-living is in its infancy $(4,34,69,120)$, these devices have been employed extensively in clinical environments to aid diagnosis and severity assessment, determine rehabilitation and intervention efficacy, and delineate pathological groups from healthy controls (Table 2). Of these applications, trunk-based IMUs are uniformly employed with the exception of a limited number of studies utilizing ear $(76,77)$, foot $(112,123)$ and smartdevice $(4,108,109)$ analysis.

\section{Diagnosis and severity assessment}

Single-point IMU gait metrics analysis has been employed as a method of assessment of ageing, orthopedic and 
neurological conditions.

Gait metrics analysis with single-point devices has aided diagnosis and severity assessment in numerous neurological diseases. Lower trunk sensor gait metrics analysis has been used to determine PD severity, demonstrating significantly reduced gait regularity and GV with increasing Hoeh and Yahr stage severity $(31,32)$. Utilizing the commercially available DynaPort, Herman et al. (57) have also classified PD subtypes based on increased gait impairment and demonstrated classification superiority based on objective gait measures compared to conventional schemes. In MS, mean spatio-temporal parameter changes have been correlated with increasing disease severity using a G-Walk sensor, while also demonstrating high correlations between gait characteristics and patient-reported outcomes, reinforcing the applicability of gait metrics analysis as a clinical measure (62). Dalton et al. (110) also demonstrated significant differences between MS severity groups based on spatio-temporal mean and variability parameters as well as autocorrelation regularity and symmetry. Determining HD severity from trunk-based gait metrics analysis has also shown to correlate with clinical scales using both linear and non-linear measures (47). In diabetes subjects, gait parameters from a trunk IMU have shown good discriminatory power in detecting those with PN in a pilot study with a small sample (111), while De Bruin et al. (53) demonstrated the discriminatory power of SL to discern PN severity in type 2 diabetes patients in free-living gait conditions.

Similarly, gait metrics analysis has aided assessment in orthopedic conditions. Using a foot IMU, Angthong \& Veljkovic (112) demonstrated significant correlations between obtained spatio-temporal parameters and subjective validated patient-reported outcomes and quality of life scores in patients with foot and ankle conditions such as arthritis, injury and tendinopathy. This is suggestive of the validity of gait assessment in clinical practice as an objective outcome measure.

In cognitive impairment, analysis of mean spatiotemporal and autocorrelation measures using a trunk IMU has been shown to predict risk of decline from mild cognitive impairment (MCI) to Alzheimer's Disease in small sample sizes $(n=23)$ (39). Furthermore, single-point gait quantification has been used to delineate between dementia subtypes (153).

Decline in physical and cognitive capacity with age is associated with frailty and disability, with consequences including falls, hospitalization and death. Numerous studies using single-point sensors and smart-devices have attempted to predict age-related gait changes $(65,108)$, predict frailty status and determine severity $(43,109)$ and predict falls and determine risk $(33,34)$ to allow early-intervention to reduce adverse outcomes.

\section{Rehabilitation and intervention efficacy}

Gait metrics analysis using single-point IMUs has been used as an objective measure of rehabilitation and intervention efficacy in a range of conditions.

Single-point IMUs have been employed in the assessment of surgical outcomes and rehabilitation. A range of objective gait measures from trunk IMUs have been obtained to determine operation efficacy and rehabilitation progress after total hip replacement (113) and decompressive laminectomy for lumbar spine stenosis (128). An ear-worn sensor has been used to evaluate recovery from anterior cruciate ligament repair based on gait symmetry (147) as well as total hip and knee replacements based on stride duration and gait symmetry in small samples (76). Similarly, employment of the consumer available Apple Watch (Apple, San Francisco, CA, USA) for detection of $\mathrm{GV}$ was claimed invaluable in monitoring recovery through continuous gait monitoring in daily life following lumbar microdiscectomy in a single patient (4).

Assessment of non-operable intervention efficacy has also been determined using trunk IMUs. In cerebral palsy subjects, improvement in spatio-temporal mean and symmetry parameters after one hippotherapy session (56) and increase in mean parameters over a twoyear intervention course (5) has been demonstrated. While linear measures of gait variability from trunk accelerations have been used to determine the effectiveness of Rivastigmine in in PD subjects over 32 weeks (114). In orthopedics, the effectiveness of prescription footwear has been assessed through trunk-based autocorrelation gait metrics analysis in severe foot and ankle fractures (115). While in women following single-breast mastectomy, significant influence of external breast prosthesis on spatiotemporal parameters of gait has been demonstrated (54).

Prescribed physical activity programs have been shown to improve physical functioning and reduce risk of falls and adverse outcomes (154). Pau et al. (60) utilized a trunk accelerometer to determine the increased effectiveness of vigorous compared to light physical activity on mean spatiotemporal parameters in elderly over 36 weeks. Similarly, the positive effect of $\mathrm{PA}$ on a range of spatio-temporal and accelerometer-based measures was demonstrated in MCI (101) and dementia subjects (38) using trunk-based 
accelerometry.

The efficacy of neurorehabilitation has also been assessed using trunk-based gait analysis. Santoyo et al. (155) demonstrated an increase in mean spatio-temporal parameters following a five-month neurorehabilitation program in 45 MS patients. Furthermore, Zanetta et al. (156) demonstrated similar improvements in cadence and GV after a four-week program, and significant correlation of gait parameters with validated clinical assessments (Berg Balance Scale, 6MWT), validating the usefulness of gait metrics analysis as an objective measure of outcomes.

\section{Delineating pathological and healthy subjects}

Most single-point IMU clinical applications focus on delineating between pathological groups with gait impairments and healthy controls. This application enables clinical validation of gait metrics and appreciation of metrics relevant to different pathologies.

Quantification of gait through single-point sensors in musculoskeletal disorders has been assessed. Knee osteoarthritis patients have been delineated from healthy controls based on numerous mean, autocorrelation and accelerometer-based spatio-temporal measures $(67,68,116)$. In amputee gait, measures of stability, regularity and variability (49) as well as mean spatio-temporal parameters (48) have shown significant difference from healthy controls. Hip adductor fatigue has been demonstrated to significantly impact variability and symmetry of gait (58), while patients with chronic lower limb pain have demonstrated significant differences in cadence, variability and symmetry measures compared to controls (74). Using a trunk IMU in pregnant patients, significant differences have also been noted in trunk movement asymmetries between those with and without lumbopelvic pain. (121)

Numerous neurological conditions show altered gait quality using single-point IMUs. In stroke, RMS, autocorrelation measures of regularity and symmetry (102) and GV (52) have shown significant differences compared to controls. The gait impairments of various ataxia disorders have been assessed, demonstrating significant differences in mean, variability and asymmetry of spatiotemporal characteristics as well as autocorrelation and accelerometer-based measures $(70,117)$. In PD, Esser et al. (46) demonstrated superior sensitivity of GV and nonlinear variability measures compared to mean parameters for delineation from healthy controls in a small sample $(n=24)$. Further works have demonstrated the ability of mean and symmetry spatio-temporal parameters as well as autocorrelation measures in both laboratory and freeliving gait to delineate between PD subjects and healthy controls $(31,69,72,118)$. Accelerometer-based measures of gait smoothness (HR) have also been shown to delineate between MS and healthy subjects prior to any measurable changes in mean spatio-temporal parameters (119), while dual-task gait in these patients has shown to result in a significant difference in mean spatio-temporal parameters compared to controls (63). In HD, significant differences have been demonstrated for autocorrelation measures, mean spatio-temporal parameters and both linear and nonlinear variability measures against healthy subjects $(47,110)$. Furthermore, in type 2 diabetes (61) and normal pressure hydrocephalus (157), altered spatio-temporal parameters have been demonstrated compared to healthy controls. In child pathological cohorts, differences in gait parameters have also been demonstrated in cerebral palsy $(42,78)$ and dyslexia subjects (122) compared to healthy controls.

The effect of cognitive impairment on gait has also been quantified compared to healthy controls using singlepoint sensors $(37,52,123,153)$. Gait differences have been quantified in those at risk or with a history of falls, with autocorrelation measures of regularity and symmetry, GV, stride length and step-time asymmetry showing significant discriminative capacity against healthy controls $(36,50)$.

\section{Limitation of single-point IMUs in delineating diseases} Clinical use of IMUs discussed above were mainly describing an obvious gait change (e.g., MS or Parkinson disease) rather than minute structural damages such as a torn hip labrum. Therefore, current use of a single-point IMU has to be considered within the appropriate clinical context. Additionally, the clinical application studies included are largely limited to straight line gait metrics assessment which does not fully reflect real-life movements. Along with the maturation of single-point IMUs in terms of validity, future studies should attempt to assess validity of these sensors in picking up complex movements that reflect real life movements such as falls.

\section{Future prospects}

The ability for wearable devices in detecting gait and posture is maturing and undergoing continuous development. Multiple studies have demonstrated the use of wearable technologies in aiding postural analysis as well as serving as a tool for the general population in everyday 
postural/activity tracking $(158,159)$. A novel scoring algorithm incorporating gait and postural scores has also been proposed to report patients' outcome in a manner which is simple and conducive to a continuous stream of data that can be remotely monitored by clinicians (160). This ability to remotely measure and record continuous data gives wearable devices an upper hand compared to lab-based instruments which are geographically sparse and perform gait analysis at discrete time points, though validity and standardization remains a drawback currently $(4,23,158-160)$.

Future studies are required before the implementation of IMUs can be recommended to clinicians. In particular, there is an urgent need to validate IMU accuracy in free-living home environments, with most current validation studies instead measuring gait metrics on straight-line pathways. Other parameters such as U-turns, complex acceleration and deceleration that mimics day-to-day movements such as slowing down when approaching a door or chair could be studied. In addition, single-point IMUs have not been consistently shown to have high accuracy when measuring variability and asymmetry metrics. Future studies may assess other models for GE detection, which are continuously being developed, that may more accurately capture these metrics. Moreover, many of the studies focusing on clinical application have described obvious gait changes. While IMUs are still useful in objectively quantifying these changes, more evidence is required to demonstrate the clinical applications of IMUs in the measurement of subtle gait differences. Future studies to compare user acceptability and compliance between single-point IMUs and multipoint IMUs should also be conducted. Studies to determine ideal placement location of single-point IMUs at various body parts could also be conducted. Further developments and validation may one day bridge the gap for incorporating wearable technologies into actual clinical setting in aiding diagnosis and monitoring patient progression.

\section{Conclusions}

This review has demonstrated the validity of single-point IMUs as an alternative to current quantitative methods and their ability to assist in clinical scenarios. The accuracy of these systems for detection of traditional metrics as well as the demonstrated clinical relevance of novel, accelerometerbased measures is promising for practicality and efficacy in the clinical context. Further validation for long-term, continuous monitoring in daily living scenarios is required as is performance in larger samples of pathological cohorts before mass commercial and clinical uptake can be expected.

\section{Acknowledgments}

Funding: None.

\section{Footnote}

Reporting Checklist: The authors have completed the PRISMA reporting checklist. Available at https://dx.doi. org/10.21037/mhealth-21-17

Conflicts of Interest: All authors have completed the ICMJE uniform disclosure form (available at https://dx.doi. org/10.21037/mhealth-21-17). The authors have no conflicts of interest to declare.

Ethical Statement: The authors are accountable for all aspects of the work in ensuring that questions related to the accuracy or integrity of any part of the work are appropriately investigated and resolved.

Open Access Statement: This is an Open Access article distributed in accordance with the Creative Commons Attribution-NonCommercial-NoDerivs 4.0 International License (CC BY-NC-ND 4.0), which permits the noncommercial replication and distribution of the article with the strict proviso that no changes or edits are made and the original work is properly cited (including links to both the formal publication through the relevant DOI and the license). See: https://creativecommons.org/licenses/by-nc-nd/4.0/.

\section{References}

1. Cook RE, Schneider I, Hazlewood ME, et al. Gait analysis alters decision-making in cerebral palsy. J Pediatr Orthop 2003;23:292-5.

2. Hodgins A, Manning O, Ritsma B, et al. Validity of Wearable Sensors in Measuring Gait Quality Following Stroke. World Stroke Congress 2018.

3. Lecat M, Decavel P, Magnin E, et al. Multiple Sclerosis and Clinical Gait Analysis before and after Fampridine: A Systematic Review. Eur Neurol 2017;78:272-86.

4. Mobbs RJ, Katsinas CJ, Choy WJ, et al. Objective monitoring of activity and Gait Velocity using wearable accelerometer following lumbar microdiscectomy to detect recurrent disc herniation. J Spine Surg 2018;4:792-7. 
5. Mutoh T, Mutoh T, Takada M, et al. Application of a triaxial accelerometry-based portable motion recorder for the quantitative assessment of hippotherapy in children and adolescents with cerebral palsy. J Phys Ther Sci 2016;28:2970-4.

6. Steultjens MP, Dekker J, van Baar ME, et al. Range of joint motion and disability in patients with osteoarthritis of the knee or hip. Rheumatology (Oxford) 2000;39:955-61.

7. Tzallas AT, Tsipouras MG, Rigas G, et al. PERFORM: a system for monitoring, assessment and management of patients with Parkinson's disease. Sensors (Basel) 2014;14:21329-57.

8. Zhang HH, Yan SH, Fang C, et al. Clinical Evaluation and Gait Characteristics before and after Total Knee Arthroplasty Based on a Portable Gait Analyzer. Orthop Surg 2016;8:360-6.

9. Verghese J, Holtzer R, Lipton RB, et al. Quantitative gait markers and incident fall risk in older adults. J Gerontol A Biol Sci Med Sci 2009;64:896-901.

10. Schwenk M, Mohler J, Wendel C, et al. Wearable sensorbased in-home assessment of gait, balance, and physical activity for discrimination of frailty status: baseline results of the Arizona frailty cohort study. Gerontology 2015;61:258-67.

11. Chen S, Lach J, Lo B, et al. Toward Pervasive Gait Analysis With Wearable Sensors: A Systematic Review. IEEE J Biomed Health Inform 2016;20:1521-37.

12. Saleh M, Murdoch G. In defence of gait analysis. Observation and measurement in gait assessment. J Bone Joint Surg Br 1985;67:237-41.

13. Cappozzo A, Della Croce U, Leardini A, et al. Human movement analysis using stereophotogrammetry. Part 1: theoretical background. Gait Posture 2005;21:186-96.

14. Spine News International. Wearables in spine surgery: Beginnings, research and real-world applications 2017. Available online: https://spinalnewsinternational.com/ wearables/

15. Rehan K. Wearing Your Spine Health on Your Sleeve Spine Universe. 2017. Available online: https://www. spineuniverse.com/treatments/emerging/wearing-yourspine-health-your-sleeve

16. Mobbs RJ, Phan K, Maharaj M, et al. Physical Activity Measured with Accelerometer and Self-Rated Disability in Lumbar Spine Surgery: A Prospective Study. Global Spine J 2016;6:459-64.

17. Phan K, Mobbs RJ. Long-Term Objective Physical Activity Measurements using a Wireless Accelerometer Following Minimally Invasive Transforaminal Interbody
Fusion Surgery. Asian Spine J 2016;10:366-9.

18. Agostini V, Gastaldi L, Rosso V, et al. A Wearable Magneto-Inertial System for Gait Analysis (H-Gait): Validation on Normal Weight and Overweight/Obese Young Healthy Adults. Sensors (Basel) 2017;17:2406.

19. Donath L, Faude O, Lichtenstein E, et al. Mobile inertial sensor based gait analysis: Validity and reliability of spatiotemporal gait characteristics in healthy seniors. Gait Posture 2016;49:371-4.

20. Kluge F, Gaßner H, Hannink J, et al. Towards Mobile Gait Analysis: Concurrent Validity and Test-Retest Reliability of an Inertial Measurement System for the Assessment of Spatio-Temporal Gait Parameters. Sensors (Basel) 2017;17:1522.

21. Maffiuletti NA, Gorelick M, Kramers-de Quervain I, et al. Concurrent validity and intrasession reliability of the IDEEA accelerometry system for the quantification of spatiotemporal gait parameters. Gait Posture 2008;27:160-3.

22. Orlowski K, Eckardt F, Herold F, et al. Examination of the reliability of an inertial sensor-based gait analysis system. Biomed Tech (Berl) 2017;62:615-22.

23. Rao PJ, Phan K, Maharaj MM, et al. Accelerometers for objective evaluation of physical activity following spine surgery. J Clin Neurosci 2016;26:14-8.

24. Barth J, Klucken J, Kugler P, et al. Biometric and mobile gait analysis for early diagnosis and therapy monitoring in Parkinson's disease. Annu Int Conf IEEE Eng Med Biol Soc 2011;2011:868-71.

25. Guo Y, Wu D, Liu G, et al. A low-cost body inertialsensing network for practical gait discrimination of hemiplegia patients. Telemed J E Health 2012;18:748-54.

26. Hsu YL, Chung PC, Wang WH, et al. Gait and balance analysis for patients with Alzheimer's disease using an inertial-sensor-based wearable instrument. IEEE J Biomed Health Inform 2014;18:1822-30.

27. Mackey AH, Stott NS, Walt SE. Reliability and validity of an activity monitor (IDEEA) in the determination of temporal-spatial gait parameters in individuals with cerebral palsy. Gait Posture 2008;28:634-9.

28. Tadano S, Takeda R, Sasaki K, et al. Gait characterization for osteoarthritis patients using wearable gait sensors (H-Gait systems). J Biomech 2016;49:684-90.

29. Tunca C, Pehlivan N, Ak N, et al. Inertial Sensor-Based Robust Gait Analysis in Non-Hospital Settings for Neurological Disorders. Sensors (Basel) 2017;17:825.

30. Sprager S, Juric MB. Inertial Sensor-Based Gait Recognition: A Review. Sensors (Basel) 2015;15:22089-127. 
31. Demonceau M, Donneau AF, Croisier JL, et al. Contribution of a Trunk Accelerometer System to the Characterization of Gait in Patients With Mild-toModerate Parkinson's Disease. IEEE J Biomed Health Inform 2015;19:1803-8.

32. Cheng JZ, Von Coelln R, Schrader K, et al. Accelerometry-based quantitative analysis of mobility in Parkinson disease. Neurology 2018;90:P3.044.

33. van Schooten KS, Pijnappels M, Rispens SM, et al. Daily-Life Gait Quality as Predictor of Falls in Older People: A 1-Year Prospective Cohort Study. PLoS One 2016;11:e0158623.

34. Del Din S, Galna B, Godfrey A, et al. Analysis of FreeLiving Gait in Older Adults With and Without Parkinson's Disease and With and Without a History of Falls: Identifying Generic and Disease-Specific Characteristics. J Gerontol A Biol Sci Med Sci 2019;74:500-6.

35. Espinosa HG, Thiel DV, Sorell M, et al. Can We Trust Inertial and Heart Rate Sensor Data from an APPLE Watch Device? Proceedings 2020;49:128.

36. Auvinet B, Berrut G, Touzard C, et al. Gait abnormalities in elderly fallers. J Aging Phys Act 2003;11:40-52.

37. Maquet D, Lekeu F, Warzee E, et al. Gait analysis in elderly adult patients with mild cognitive impairment and patients with mild Alzheimer's disease: simple versus dual task: a preliminary report. Clin Physiol Funct Imaging 2010;30:51-6.

38. Perrochon A, Tchalla AE, Bonis J, et al. Effects of a Multicomponent Exercise Program on Spatiotemporal Gait Parameters, Risk of Falling and Physical Activity in Dementia Patients. Dement Geriatr Cogn Dis Extra 2015;5:350-60.

39. Gillain S, Dramé M, Lekeu F, et al. Gait speed or gait variability, which one to use as a marker of risk to develop Alzheimer disease? A pilot study. Aging Clin Exp Res 2016;28:249-55.

40. Walking and running analysis gait analysis devices Paris: Centaure Metrix. [cited 2021 Jun 28]. Available online: http://www.centaure-metrix.com/Index_en.html

41. Esser P, Dawes H, Collett J, et al. Assessment of spatiotemporal gait parameters using inertial measurement units in neurological populations. Gait Posture 2011;34:558-60.

42. Saether R, Helbostad JL, Adde L, et al. Gait characteristics in children and adolescents with cerebral palsy assessed with a trunk-worn accelerometer. Res Dev Disabil 2014;35:1773-81.

43. Martínez-Ramírez A, Martinikorena I, Gómez M, et al. Frailty assessment based on trunk kinematic parameters during walking. J Neuroeng Rehabil 2015;12:48.

44. Martínez-Ramírez A, Martinikorena I, Lecumberri P, et al. Dual Task Gait Performance in Frail Individuals with and without Mild Cognitive Impairment. Dement Geriatr Cogn Disord 2016;42:7-16.

45. MTi-1 IMU Enschede: Xsens. [cited 2021 Jun 28]. Available online: https://www.xsens.com/mti-1-imu

46. Esser P, Dawes H, Collett J, et al. Insights into gait disorders: walking variability using phase plot analysis, Parkinson's disease. Gait Posture 2013;38:648-52.

47. Collett J, Esser P, Khalil H, et al. Insights into gait disorders: walking variability using phase plot analysis, Huntington's disease. Gait Posture 2014;40:694-700.

48. Houdijk H, Appelman FM, Van Velzen JM, et al. Validity of DynaPort GaitMonitor for assessment of spatiotemporal parameters in amputee gait. J Rehabil Res Dev 2008;45:1335-42.

49. Lamoth CJ, Ainsworth E, Polomski W, et al. Variability and stability analysis of walking of transfemoral amputees. Med Eng Phys 2010;32:1009-14.

50. Bautmans I, Jansen B, Van Keymolen B, et al. Reliability and clinical correlates of 3D-accelerometry based gait analysis outcomes according to age and fall-risk. Gait Posture 2011;33:366-72.

51. Lamoth CJ, van Deudekom FJ, van Campen JP, et al. Gait stability and variability measures show effects of impaired cognition and dual tasking in frail people. J Neuroeng Rehabil 2011;8:2.

52. Meijer R, Plotnik M, Zwaaftink EG, et al. Markedly impaired bilateral coordination of gait in post-stroke patients: Is this deficit distinct from asymmetry? A cohort study. J Neuroeng Rehabil 2011;8:23.

53. de Bruin ED, Hubli M, Hofer P, et al. Validity and reliability of accelerometer-based gait assessment in patients with diabetes on challenging surfaces. J Aging Res 2012;2012:954378.

54. Hojan K, Manikowska F, Molinska-Glura M, et al. The impact of an external breast prosthesis on the gait parameters of women after mastectomy. Cancer Nurs 2014;37:E30-6.

55. Manikowska F, Hojan K, Chen PJ, et al. The gait pattern in post-menopausal women. Pilot study. Ortop Traumatol Rehabil 2013;15:575-83.

56. Manikowska F, Jóźwiak M, Idzior M, et al. The effect of a hippotherapy session on spatiotemporal parameters of gait in children with cerebral palsy - pilot study. Ortop Traumatol Rehabil 2013;15:253-7.

57. Herman T, Weiss A, Brozgol M, et al. Gait and balance 
in Parkinson's disease subtypes: objective measures and classification considerations. J Neurol 2014;261:2401-10.

58. Arvin M, Hoozemans MJ, Burger BJ, et al. Effects of hip abductor muscle fatigue on gait control and hip position sense in healthy older adults. Gait Posture 2015;42:545-9.

59. MoveMonitor The Hague: McRoberts. [cited 2021 Jun 28]. Available online: https://www.mcroberts.nl/products/ movemonitor/

60. Pau M, Leban B, Collu G, et al. Effect of light and vigorous physical activity on balance and gait of older adults. Arch Gerontol Geriatr 2014;59:568-73.

61. Awotidebe TO, Ativie RN, Oke KI, et al. Relationships among exercise capacity, dynamic balance and gait characteristics of Nigerian patients with type-2 diabetes: an indication for fall prevention. J Exerc Rehabil 2016;12:581-8.

62. Pau M, Caggiari S, Mura A, et al. Clinical assessment of gait in individuals with multiple sclerosis using wearable inertial sensors: Comparison with patient-based measure. Mult Scler Relat Disord 2016;10:187-91.

63. Pau M, Corona F, Pilloni G, et al. Texting while walking differently alters gait patterns in people with multiple sclerosis and healthy individuals. Mult Scler Relat Disord 2018;19:129-33.

64. G-Walk: Wearable system for the functional analysis of movement Milan: BTS Bioengineering. [cited 2021 Jun 28]. Available online: https://www.btsbioengineering.com/ products/g-walk-inertial-motion-system/

65. Terrier P, Reynard F. Effect of age on the variability and stability of gait: a cross-sectional treadmill study in healthy individuals between 20 and 69 years of age. Gait Posture 2015;41:170-4.

66. Compact and versatile wireless inertial measurement unit Lausanne: Gaitup. [cited 2021 Jun 28]. Available online: https://research.gaitup.com/physilog/

67. Barden JM, Clermont CA, Kobsar D, et al. AccelerometerBased Step Regularity Is Lower in Older Adults with Bilateral Knee Osteoarthritis. Front Hum Neurosci 2016;10:625.

68. Clermont CA, Barden JM. Accelerometer-based determination of gait variability in older adults with knee osteoarthritis. Gait Posture 2016;50:126-30.

69. Del Din S, Godfrey A, Galna B, et al. Free-living gait characteristics in ageing and Parkinson's disease: impact of environment and ambulatory bout length. J Neuroeng Rehabil 2016;13:46.

70. Hickey A, Gunn E, Alcock L, et al. Validity of a wearable accelerometer to quantify gait in spinocerebellar ataxia type 6. Physiol Meas 2016;37:N105-17.

71. AX3 York: Axivity. [cited 2021 Jun 28]. Available online: https://axivity.com/product/ax3

72. Hatanaka N, Sato K, Hishikawa N, et al. Comparative Gait Analysis in Progressive Supranuclear Palsy and Parkinson's Disease. Eur Neurol 2016;75:282-9.

73. Edwardson CL, Winkler EAH, Bodicoat DH, et al. Considerations when using the activPAL monitor in fieldbased research with adult populations. J Sport Health Sci 2017;6:162-78.

74. Terrier P, Le Carre J, Connaissa ML, et al. Monitoring of Gait Quality in Patients With Chronic Pain of Lower Limbs. IEEE Trans Neural Syst Rehabil Eng 2017;25:1843-52.

75. ActiGraph wGT3X-BT. Pensacola: Actigraph. [cited 2021 Jun 28]. Available online: https://actigraphcorp.com/ actigraph-wgt $3 \mathrm{x}-\mathrm{bt} /$

76. Atallah L, Wiik A, Lo B, et al. Gait asymmetry detection in older adults using a light ear-worn sensor. Physiol Meas 2014;35:N29-40.

77. Jarchi D, Lo B, Wong C, et al. Gait Analysis From a Single Ear-Worn Sensor: Reliability and Clinical Evaluation for Orthopaedic Patients. IEEE Trans Neural Syst Rehabil Eng 2016;24:882-92.

78. Iosa M, Morelli D, Marro T, et al. Ability and stability of running and walking in children with cerebral palsy. Neuropediatrics 2013;44:147-54.

79. Potter MV, Ojeda LV, Perkins NC, et al. Effect of IMU Design on IMU-Derived Stride Metrics for Running. Sensors (Basel) 2019;19:2601.

80. Felisberto F, Fdez-Riverola F, Pereira A. A ubiquitous and low-cost solution for movement monitoring and accident detection based on sensor fusion. Sensors (Basel) 2014;14:8961-83.

81. Perry J, Schoneberger B. Gait Analysis: Normal and Pathological Function. SLACK, 1992.

82. Lord S, Galna B, Verghese J, et al. Independent domains of gait in older adults and associated motor and nonmotor attributes: validation of a factor analysis approach. J Gerontol A Biol Sci Med Sci 2013;68:820-7.

83. Lord S, Galna B, Coleman S, et al. Mild depressive symptoms are associated with gait impairment in early Parkinson's disease. Mov Disord 2013;28:634-9.

84. Beauchet O, Allali G, Sekhon H, et al. Guidelines for Assessment of Gait and Reference Values for Spatiotemporal Gait Parameters in Older Adults: The Biomathics and Canadian Gait Consortiums Initiative. Front Hum Neurosci 2017;11:353. 
85. McCamley J, Donati M, Grimpampi E, et al. An enhanced estimate of initial contact and final contact instants of time using lower trunk inertial sensor data. Gait Posture 2012;36:316-8.

86. Zijlstra W, Hof AL. Assessment of spatio-temporal gait parameters from trunk accelerations during human walking. Gait Posture 2003;18:1-10.

87. González RC, Alvarez D, López AM, et al. Ambulatory estimation of mean step length during unconstrained walking by means of COG accelerometry. Comput Methods Biomech Biomed Engin 2009;12:721-6.

88. Köse A, Cereatti A, Della Croce U. Bilateral step length estimation using a single inertial measurement unit attached to the pelvis. J Neuroeng Rehabil 2012;9:9.

89. Shin SH, Park CG. Adaptive step length estimation algorithm using optimal parameters and movement status awareness. Med Eng Phys 2011;33:1064-71.

90. Li Q, Young M, Naing V, et al. Walking speed estimation using a shank-mounted inertial measurement unit. J Biomech 2010;43:1640-3.

91. Sabatini AM, Martelloni C, Scapellato S, et al. Assessment of walking features from foot inertial sensing. IEEE Trans Biomed Eng 2005;52:486-94.

92. Song M, Kim J. An Ambulatory Gait Monitoring System with Activity Classification and Gait Parameter Calculation Based on a Single Foot Inertial Sensor. IEEE Trans Biomed Eng 2018;65:885-93.

93. Baroudi L, Newman MW, Jackson EA, et al. Estimating Walking Speed in the Wild. Front Sports Act Living 2020;2:583848.

94. Moe-Nilssen R, Helbostad JL. Estimation of gait cycle characteristics by trunk accelerometry. J Biomech 2004;37:121-6.

95. Godfrey A, Del Din S, Barry G, et al. Instrumenting gait with an accelerometer: a system and algorithm examination. Med Eng Phys 2015;37:400-7.

96. Sekine M, Akay M, Tamura T, et al. Fractal dynamics of body motion in patients with Parkinson's disease. J Neural Eng 2004;1:8-15.

97. Akay M, Sekine M, Tamura T, et al. Fractal dynamics of body motion in post-stroke hemiplegic patients during walking. J Neural Eng 2004;1:111-6.

98. Hausdorff JM. Gait dynamics in Parkinson's disease: common and distinct behavior among stride length, gait variability, and fractal-like scaling. Chaos 2009;19:026113.

99. Rochester L, Chastin SF, Lord S, et al. Understanding the impact of deep brain stimulation on ambulatory activity in advanced Parkinson's disease. J Neurol 2012;259:1081-6.
100.Brach JS, McGurl D, Wert D, et al. Validation of a measure of smoothness of walking. J Gerontol A Biol Sci Med Sci 2011;66:136-41.

101. Doi T, Makizako H, Shimada H, et al. Effects of multicomponent exercise on spatial-temporal gait parameters among the elderly with amnestic mild cognitive impairment (aMCI): preliminary results from a randomized controlled trial (RCT). Arch Gerontol Geriatr 2013;56:104-8.

102. Mizuike C, Ohgi S, Morita S. Analysis of stroke patient walking dynamics using a tri-axial accelerometer. Gait Posture 2009;30:60-4.

103. Moher D, Liberati A, Tetzlaff J, et al. Preferred reporting items for systematic reviews and meta-analyses: the PRISMA statement. BMJ 2009;339:b2535.

104. Scottish Intercollegiate Guidelines Network (SIGN). Methodology Checklist 5: Studies of Diagnostic Accuracy. 2014. Available online: https://www.sign.ac.uk/what-wedo/methodology/checklists/

105.NHMRC. Guidelines for Guidelines: Assessing risk of bias. 2019. Available online: https://nhmrc.gov.au/ guidelinesforguidelines/develop/assessing-risk-bias

106. GA Wells, B Shea, D O'Connell, et al. The NewcastleOttawa Scale (NOS) for assessing the quality of nonrandomised studies in meta-analyses. 1999. Available online: http://www.ohri.ca/programs/clinical_ epidemiology/oxford.asp

107. Scottish Intercollegiate Guidelines Network (SIGN). Methodology checklist 2: randomised controlled trials 2014. Available online: https://www.sign.ac.uk/what-wedo/methodology/checklists/

108. Kosse NM, Vuillerme N, Hortobágyi T, et al. Multiple gait parameters derived from iPod accelerometry predict age-related gait changes. Gait Posture 2016;46:112-7.

109. Soangra R, Lockhart TE. Inertial Sensor-Based Variables Are Indicators of Frailty and Adverse Post-Operative Outcomes in Cardiovascular Disease Patients. Sensors (Basel) 2018;18:1792.

110. Dalton A, Khalil H, Busse M, et al. Analysis of gait and balance through a single triaxial accelerometer in presymptomatic and symptomatic Huntington's disease. Gait Posture 2013;37:49-54.

111.Esser P, Collett J, Maynard K, et al. Single Sensor Gait Analysis to Detect Diabetic Peripheral Neuropathy: A Proof of Principle Study. Diabetes Metab J 2018;42:82-6.

112.Angthong C, Veljkovic A. Relationships among subjective patient-reported outcome, quality of life, and objective gait characteristics using wearable foot inertial-sensor 
assessment in foot-ankle patients. Eur J Orthop Surg Traumatol 2019;29:683-7.

113. Rapp W, Brauner T, Weber L, et al. Improvement of walking speed and gait symmetry in older patients after hip arthroplasty: a prospective cohort study. BMC Musculoskelet Disord 2015;16:291.

114.Henderson EJ, Lord SR, Brodie MA, et al. Rivastigmine for gait stability in patients with Parkinson's disease (ReSPonD): a randomised, double-blind, placebocontrolled, phase 2 trial. Lancet Neurol 2016;15:249-58.

115. Terrier P, Dériaz O, Meichtry A, et al. Prescription footwear for severe injuries of foot and ankle: effect on regularity and symmetry of the gait assessed by trunk accelerometry. Gait Posture 2009;30:492-6.

116. Bolink SA, van Laarhoven SN, Lipperts $M$, et al. Inertial sensor motion analysis of gait, sit-stand transfers and stepup transfers: differentiating knee patients from healthy controls. Physiol Meas 2012;33:1947-58.

117. Matsushima A, Yoshida K, Genno H, et al. Clinical assessment of standing and gait in ataxic patients using a triaxial accelerometer. Cerebellum Ataxias 2015;2:9.

118. Yang CC, Hsu YL, Shih KS, et al. Real-time gait cycle parameter recognition using a wearable accelerometry system. Sensors (Basel) 2011;11:7314-26.

119.Pau M, Mandaresu S, Pilloni G, et al. Smoothness of gait detects early alterations of walking in persons with multiple sclerosis without disability. Gait Posture 2017;58:307-9.

120.Storm FA, Nair KPS, Clarke AJ, et al. Free-living and laboratory gait characteristics in patients with multiple sclerosis. PLoS One 2018;13:e0196463.

121. Tanigawa A, Morino S, Aoyama T, et al. Gait analysis of pregnant patients with lumbopelvic pain using inertial sensor. Gait Posture 2018;65:176-81.

122. Moe-Nilssen R, Helbostad JL, Talcott JB, et al. Balance and gait in children with dyslexia. Exp Brain Res 2003;150:237-44.

123. Chung P, Hsu Y, Wang C, et al. Gait analysis for patients with Alzheimer'S disease using a triaxial accelerometer. 2012 IEEE International Symposium on Circuits and Systems (ISCAS), 2012:1323-6.

124. Bugané F, Benedetti MG, Casadio G, et al. Estimation of spatial-temporal gait parameters in level walking based on a single accelerometer: validation on normal subjects by standard gait analysis. Comput Methods Programs Biomed 2012;108:129-37.

125. Hartmann A, Luzi S, Murer K, et al. Concurrent validity of a trunk tri-axial accelerometer system for gait analysis in older adults. Gait Posture 2009;29:444-8.
126. Hartmann A, Murer K, de Bie RA, et al. Reproducibility of spatio-temporal gait parameters under different conditions in older adults using a trunk tri-axial accelerometer system. Gait Posture 2009;30:351-5.

127. Byun S, Han JW, Kim TH, et al. Test-Retest Reliability and Concurrent Validity of a Single Tri-Axial Accelerometer-Based Gait Analysis in Older Adults with Normal Cognition. PLoS One 2016;11:e0158956.

128. Ohtaki Y, Mamizuka N, Hirano A, et al. Pre- and Postoperative Evaluations of Patients with Lumbar Spinal Stenosis by Clinical Walk Test Using an Inertial Sensor. Transactions of Japanese Society for Medical and Biological Engineering 2014;52:167-74.

129. Jarchi D, Pope J, Lee TKM, et al. A Review on Accelerometry-Based Gait Analysis and Emerging Clinical Applications. IEEE Rev Biomed Eng 2018;11:177-94.

130. Brandes M, Zijlstra W, Heikens S, et al. Accelerometry based assessment of gait parameters in children. Gait Posture 2006;24:482-6.

131.Zijlstra A, Zijlstra W. Trunk-acceleration based assessment of gait parameters in older persons: a comparison of reliability and validity of four inverted pendulum based estimations. Gait Posture 2013;38:940-4.

132. Esser P, Dawes H, Collett J, et al. Validity and inter-rater reliability of inertial gait measurements in Parkinson's disease: a pilot study. J Neurosci Methods 2012;205:177-81.

133. De Ridder R, Lebleu J, Willems T, et al. Concurrent Validity of a Commercial Wireless Trunk Triaxial Accelerometer System for Gait Analysis. J Sport Rehabil 2019;28:jsr.

134. Lim SY, Lee WH. Comparison of accelerometer-based and treadmill-based analysis systems for measuring gait parameters in healthy adults. J Phys Ther Sci 2017;29:651-3.

135.Park G; BHSc; Woo Y. Comparison between a center of mass and a foot pressure sensor system for measuring gait parameters in healthy adults. J Phys Ther Sci 2015;27:3199-202.

136.Zago M, Sforza C, Pacifici I, et al. Gait evaluation using inertial measurement units in subjects with Parkinson's disease. J Electromyogr Kinesiol 2018;42:44-8.

137. Gudesblatt M, Trebing S, Burke C, et al. Multiple sclerosis, gait and digital devices: A comparison of two FDA approved validated devices that provide multidimensional quantifiable gait parameters in people with multiple sclerosis (PWMS). Neurology Conference: 70th Annual Meeting of the American Academy of Neurology, AAN. 2018;90: P4.400.

138. Henriksen M, Lund H, Moe-Nilssen R, et al. Test-retest 
reliability of trunk accelerometric gait analysis. Gait Posture 2004;19:288-97.

139. Grimpampi E, Oesen S, Halper B, et al. Reliability of gait variability assessment in older individuals during a sixminute walk test. J Biomech 2015;48:4185-9.

140.Storm FA, Buckley CJ, Mazzà C. Gait event detection in laboratory and real life settings: Accuracy of ankle and waist sensor based methods. Gait Posture 2016;50:42-6.

141.Godfrey A, Del Din S, Barry G, et al. Within trial validation and reliability of a single tri-axial accelerometer for gait assessment. Annu Int Conf IEEE Eng Med Biol Soc 2014;2014:5892-5.

142.González RC, López AM, Rodriguez-Uría J, et al. Realtime gait event detection for normal subjects from lower trunk accelerations. Gait Posture 2010;31:322-5.

143. Trojaniello D, Cereatti A, Della Croce U. Accuracy, sensitivity and robustness of five different methods for the estimation of gait temporal parameters using a single inertial sensor mounted on the lower trunk. Gait Posture 2014;40:487-92.

144. Trojaniello D, Ravaschio A, Hausdorff JM, et al. Comparative assessment of different methods for the estimation of gait temporal parameters using a single inertial sensor: application to elderly, post-stroke, Parkinson's disease and Huntington's disease subjects. Gait Posture 2015;42:310-6.

145. Oyake K, Yamaguchi T, Sugasawa M, et al. Validity of gait asymmetry estimation by using an accelerometer in individuals with hemiparetic stroke. J Phys Ther Sci 2017;29:307-11.

146. Sejdić E, Lowry KA, Bellanca J, et al. Extraction of stride events from gait accelerometry during treadmill walking. IEEE J Transl Eng Health Med 2016. doi: 10.1109/ JTEHM.2015.2504961.

147.Jarchi D, Lo B, Ieong E, et al. Validation of the e-AR Sensor for Gait Event Detection Using the Parotec Foot Insole with Application to Post-Operative Recovery Monitoring. 2014 11th International Conference on Wearable and Implantable Body Sensor Networks; 2014:127-31.

148.Jarchi D, Peters A, Lo B, et al. Assessment of the e-AR sensor for gait analysis of Parkinson;s Disease patients. 2015 IEEE 12th International Conference on Wearable and Implantable Body Sensor Networks (BSN). 2015:1-6.

149. Maqbool HF, Husman MAB, Awad MI, et al. A Real-Time Gait Event Detection for Lower Limb Prosthesis Control and Evaluation. IEEE Trans Neural Syst Rehabil Eng 2017;25:1500-9.
150.Lemoyne R, Mastroianni T, Cozza M, et al. Implementation of an iPhone as a wireless accelerometer for quantifying gait characteristics. Annu Int Conf IEEE Eng Med Biol Soc 2010;2010:3847-51.

151.Pepa L, Verdini F, Spalazzi L. Gait parameter and event estimation using smartphones. Gait Posture 2017;57:217-23.

152. Silsupadol P, Teja K, Lugade V. Reliability and validity of a smartphone-based assessment of gait parameters across walking speed and smartphone locations: Body, bag, belt, hand, and pocket. Gait Posture 2017;58:516-22.

153. Ardle RM, Galna B, Thomas A, et al. Continuous Monitoring of Gait: What Can It Tell Us About Dementia? Alzheimer's and Dementia 2018;14:P192-3.

154. Suttanon P, Hill KD, Said CM, et al. Feasibility, safety and preliminary evidence of the effectiveness of a home-based exercise programme for older people with Alzheimer's disease: a pilot randomized controlled trial. Clin Rehabil 2013;27:427-38.

155. Santoyo C, Fabregas D, Janer M, et al. Changes in spatiotemporal gait parameters after physical rehabilitation in Multiple Sclerosis: A descriptive analysis. Mult Scler J 2018;24:866.

156.Zanetta C, Pisa M, Guerrieri S, et al. Intensive neurorehabilitation is associated with improved gait kinematic analysis in progressive multiple sclerosis. Mult Scler J 2017;23:144.

157. Ono Y, Ora H, Kiko Y, et al. Gait evaluation of normal pressure hydrocephalus using inertial sensor. J Neurol Sci 2017;381:722.

158. Chakravorty A, Mobbs RJ, Anderson DB, et al. The role of wearable devices and objective gait analysis for the assessment and monitoring of patients with lumbar spinal stenosis: systematic review. BMC Musculoskelet Disord 2019;20:288.

159. Yoong NKM, Perring J, Mobbs RJ. Commercial Postural Devices: A Review. Sensors (Basel) 2019;19:5128.

160.Simpson L, Maharaj MM, Mobbs RJ. The role of wearables in spinal posture analysis: a systematic review. BMC Musculoskelet Disord 2019;20:55.

doi: $10.21037 /$ mhealth-21-17

Cite this article as: Mobbs RJ, Perring J, Raj SM, Maharaj M, Yoong NKM, Sy LW, Fonseka RD, Natarajan P, Choy WJ. Gait metrics analysis utilizing single-point inertial measurement units: a systematic review. mHealth 2022;8:9. 


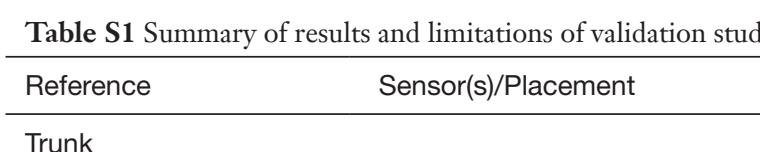

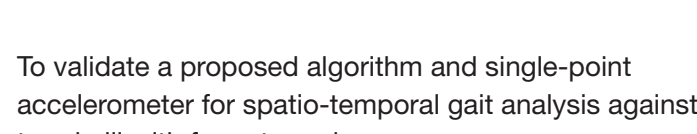

and

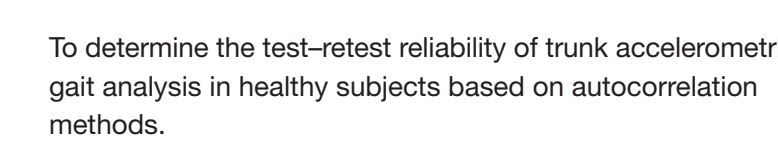

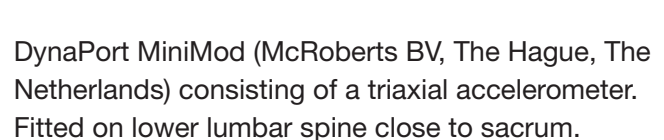

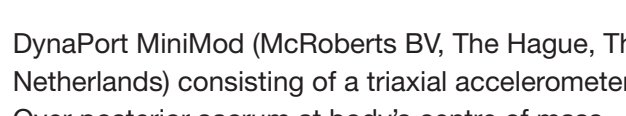

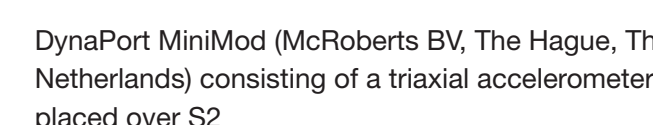

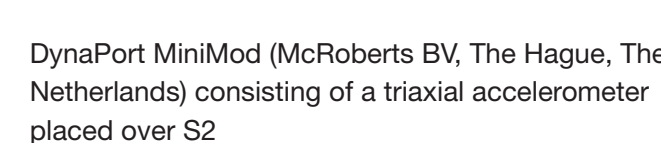

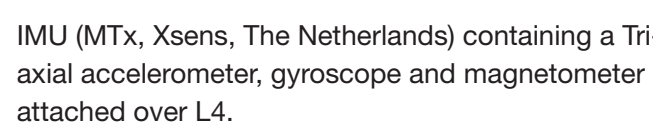

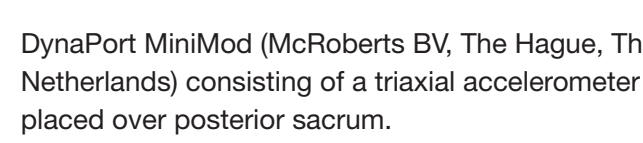

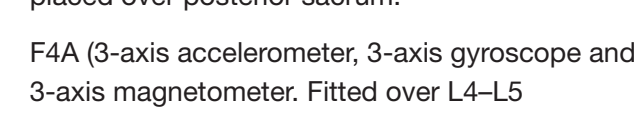

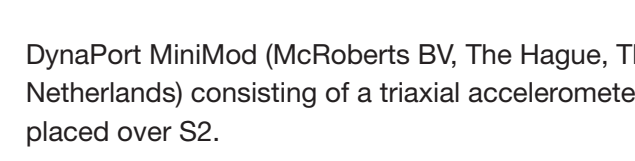

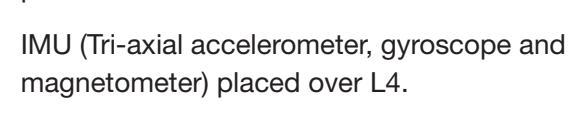

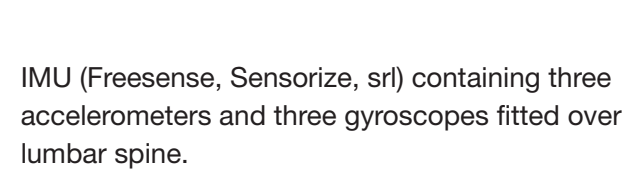

AD.BBC Sensor
uppers sterum.

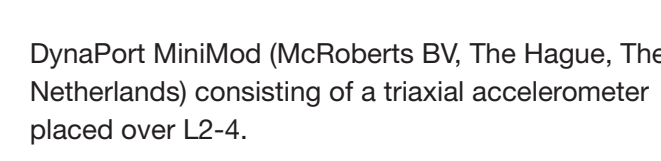

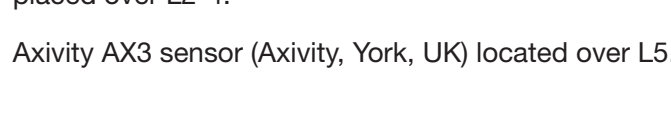

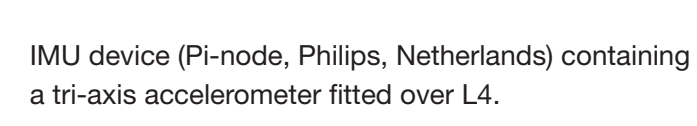

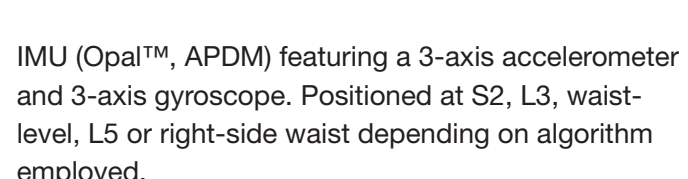

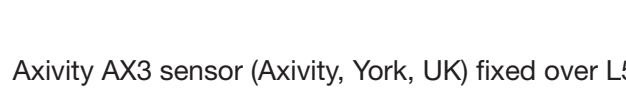

IIMU (Fresesense, Sens
lower lumbar spine.

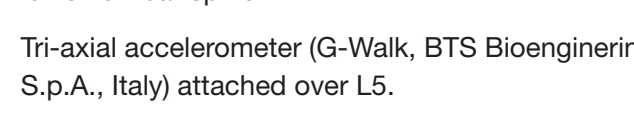

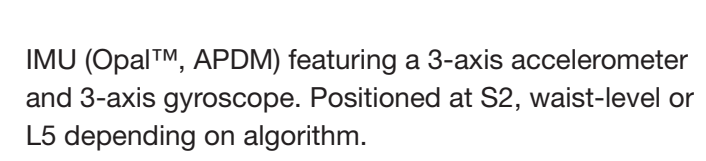

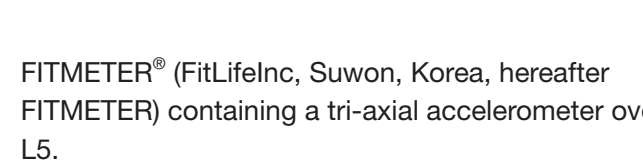

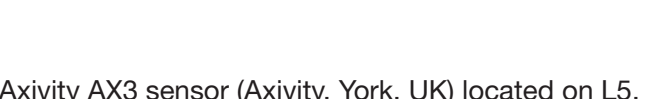

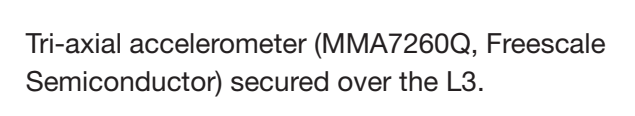

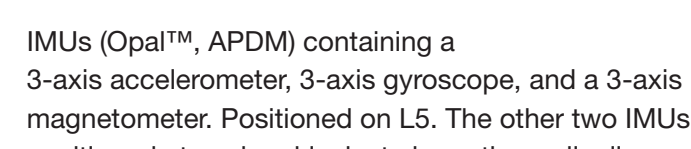

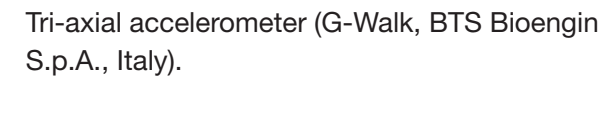

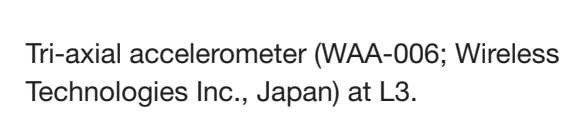

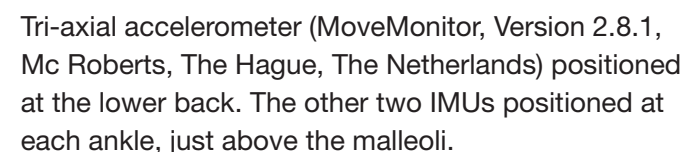

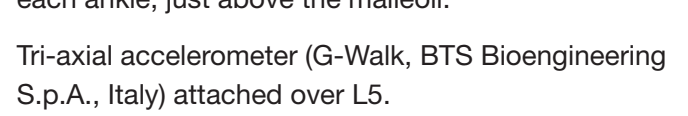

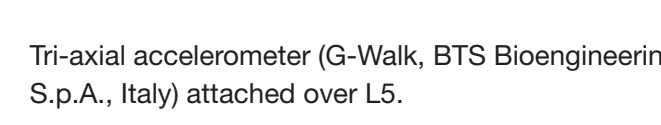

Waist

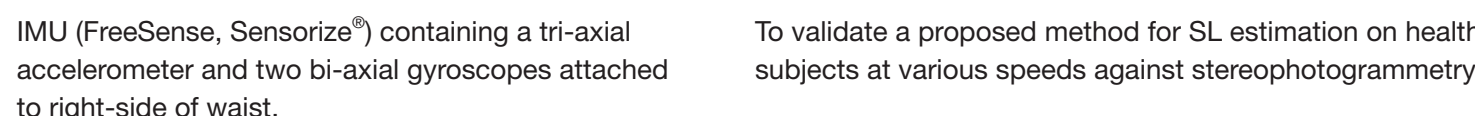

Ide length, SL Land cadence.

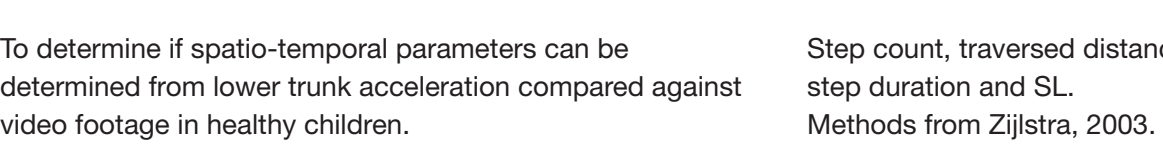

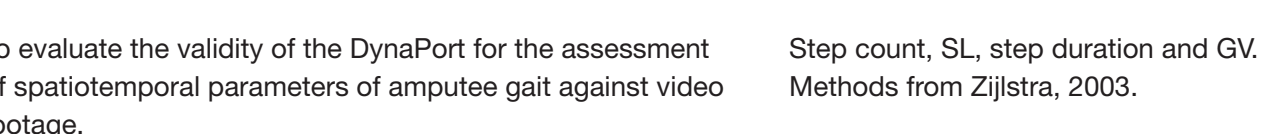

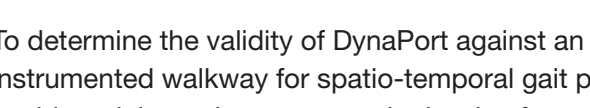

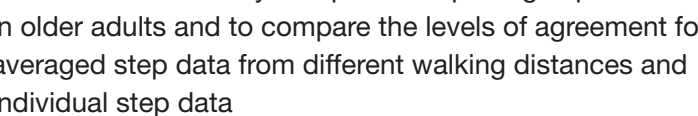

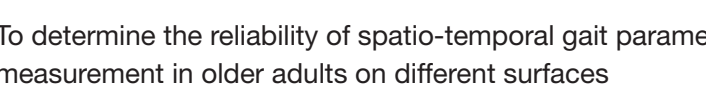

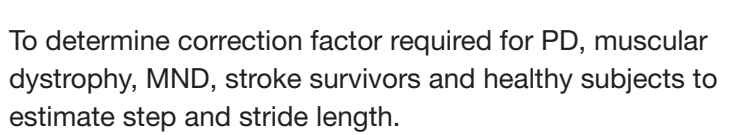

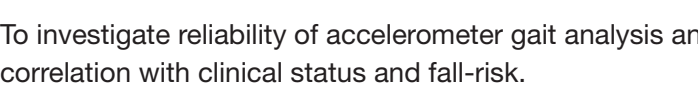

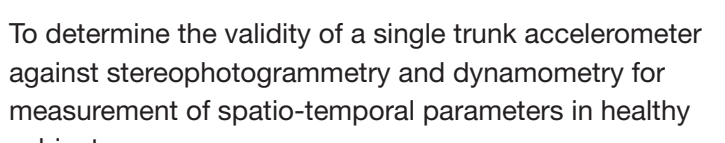

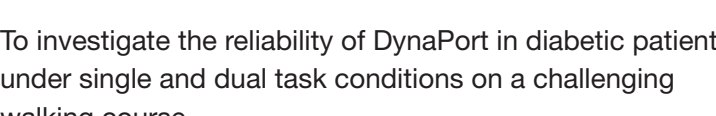

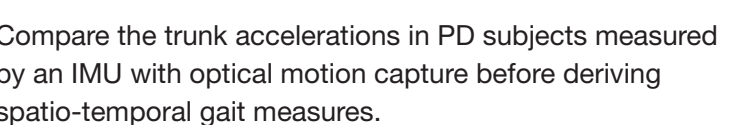

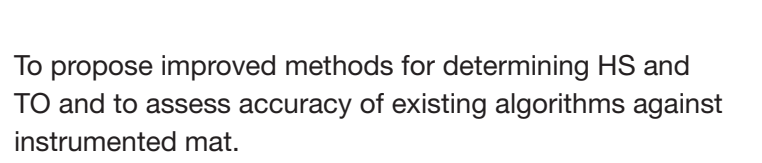

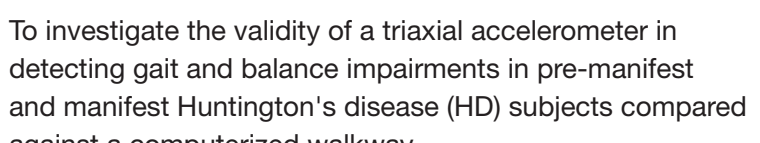

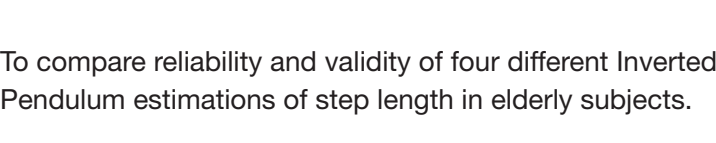

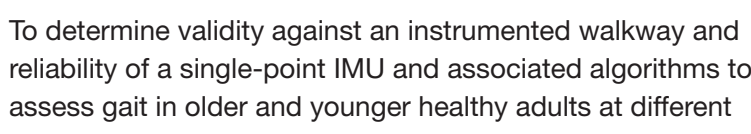

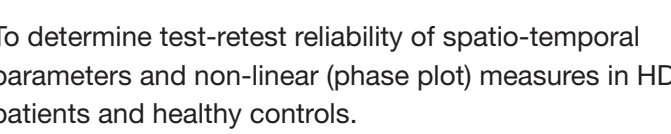

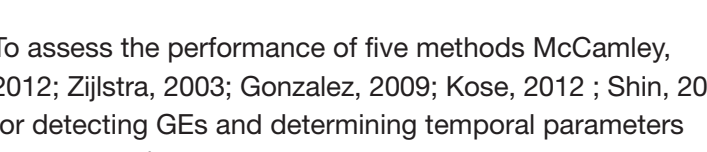

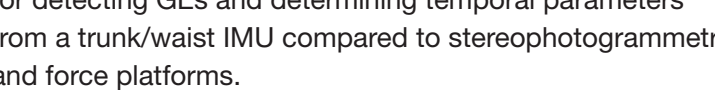

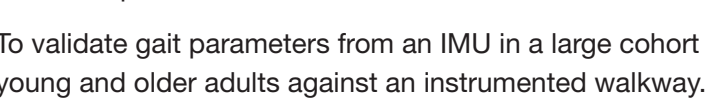

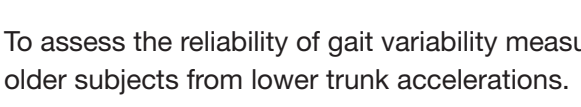

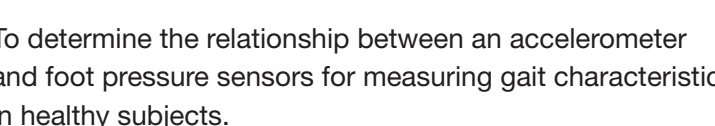

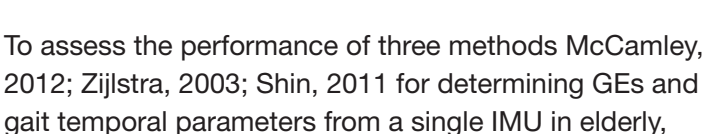

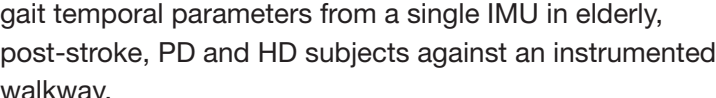

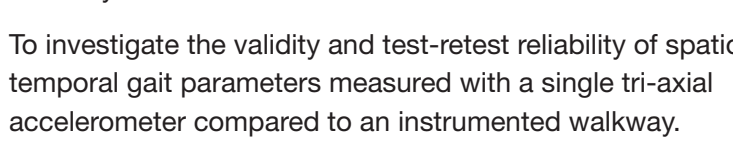

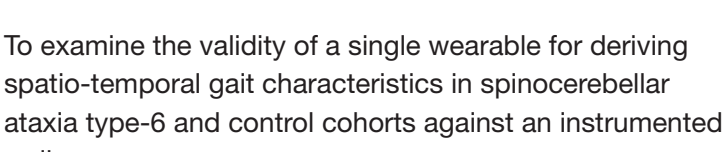

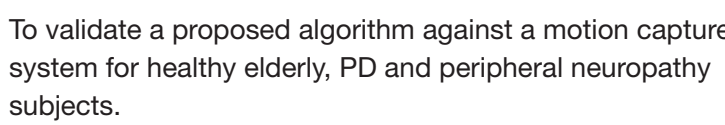

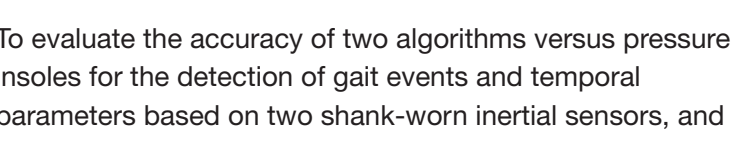

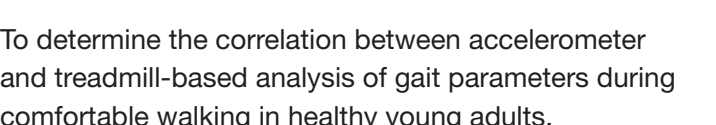

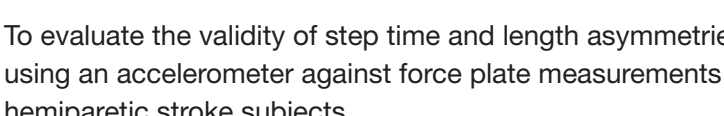

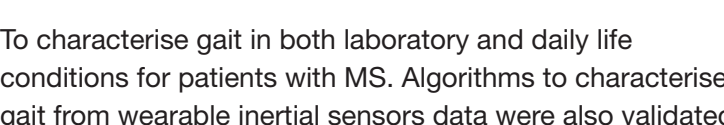

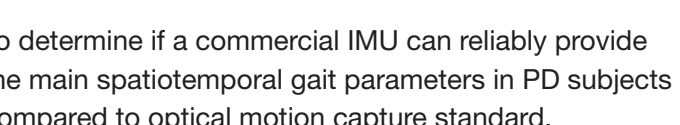

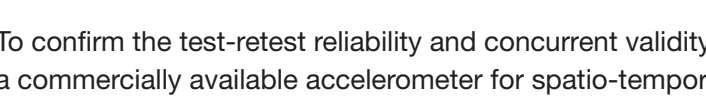

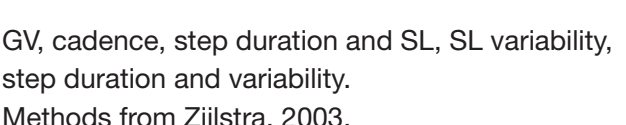

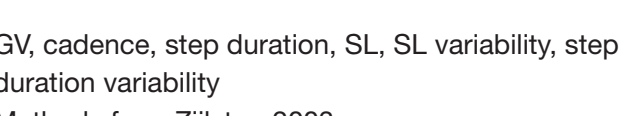

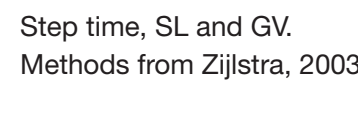

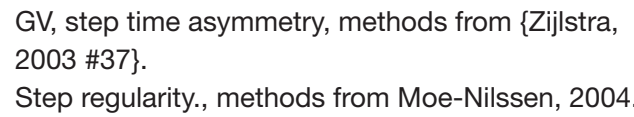

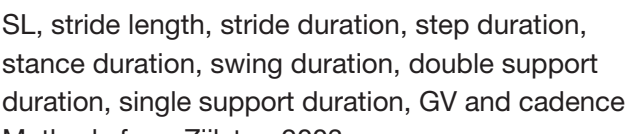

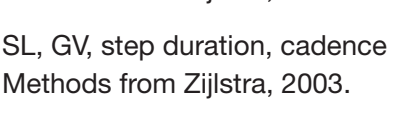
Stride length, GV. Step duration.
Methods from Zilistra, 2003. HS., TO and stride duration.
Methods from Mccamley, 2012.

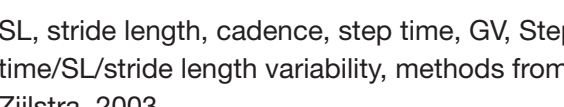

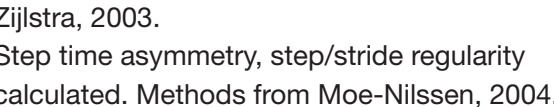

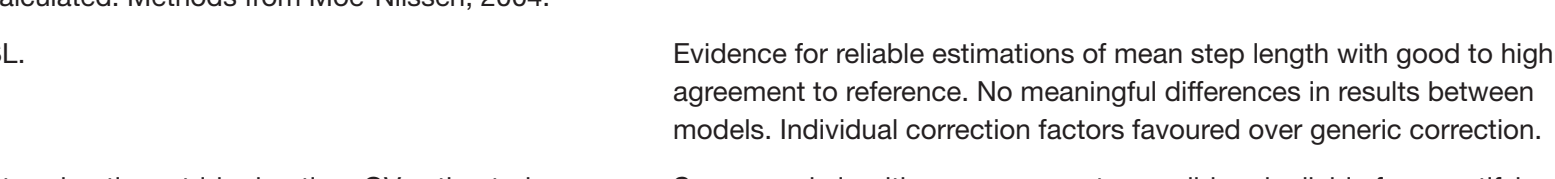

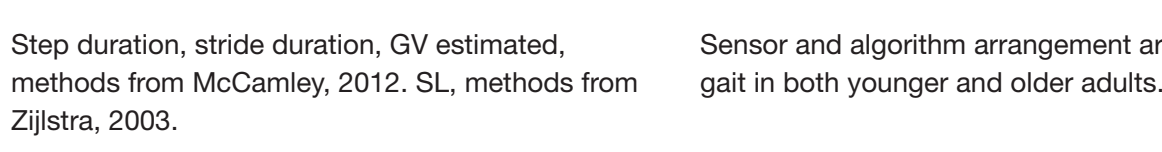

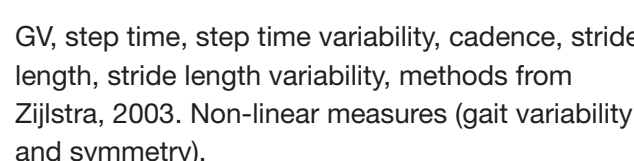

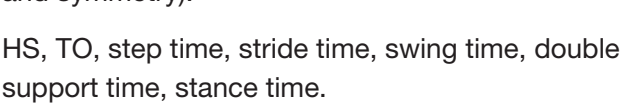

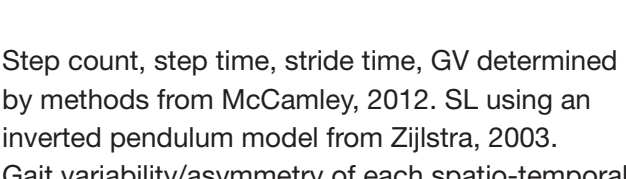

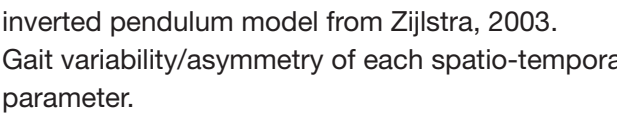

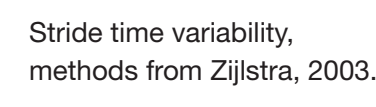

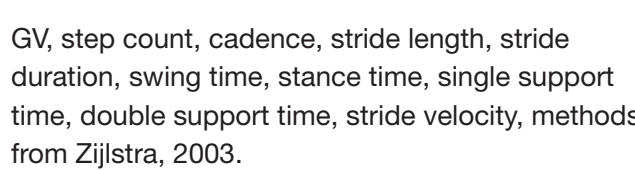

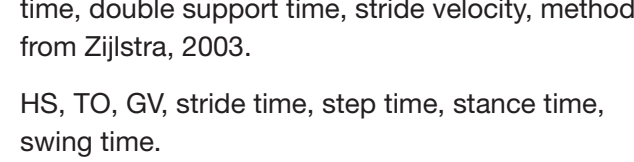

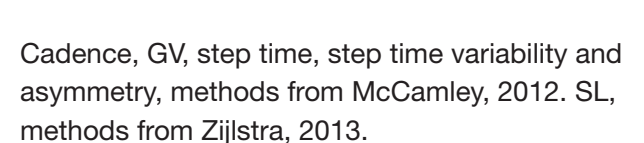

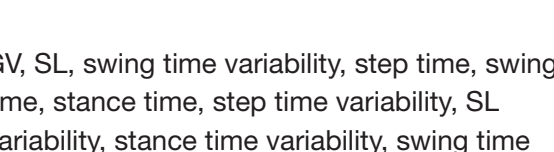

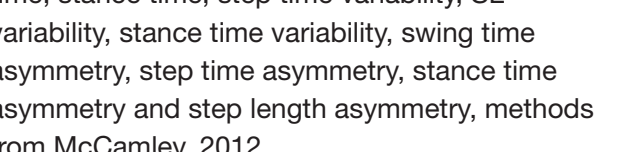

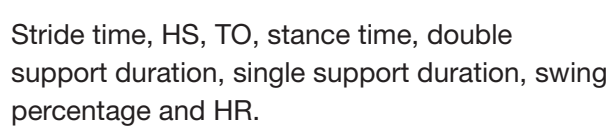

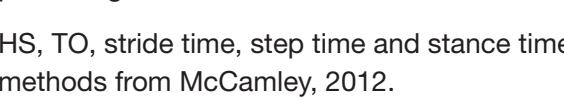

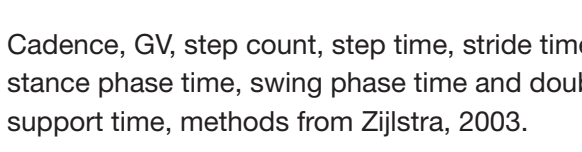

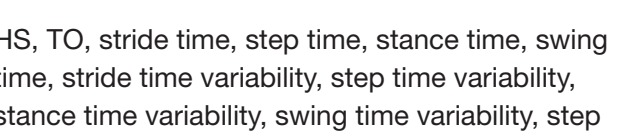

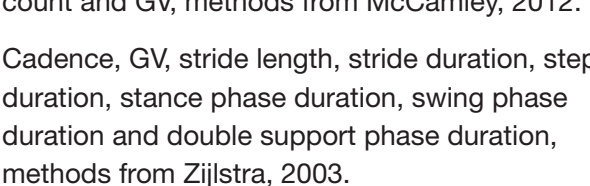

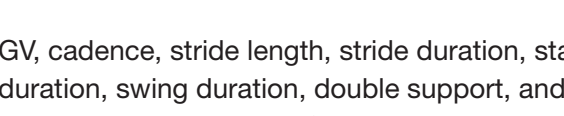

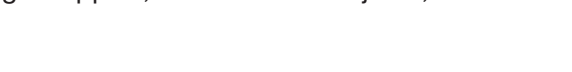

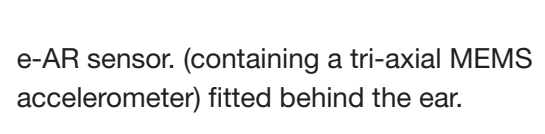

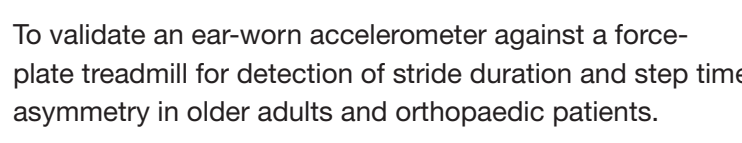

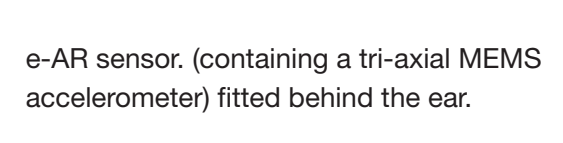

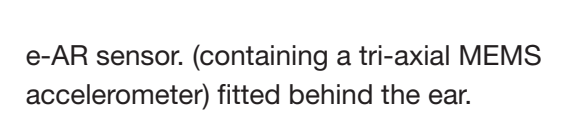

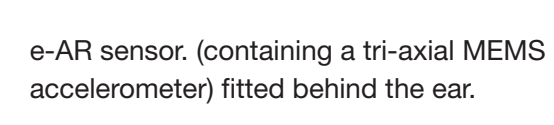

IMU (bi-axial accelerometer and
to the lateral aspect of the calf.

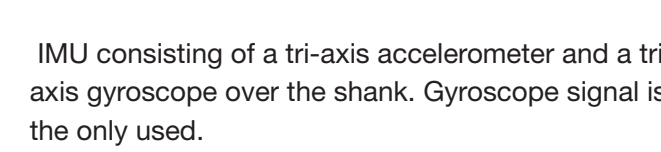

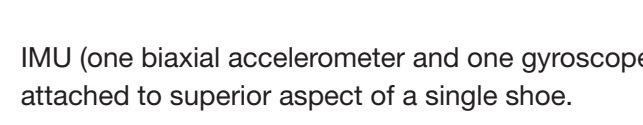

ImU (tri-ixixa acceleromete
the rarar of a single shoe. iphonescing

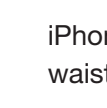

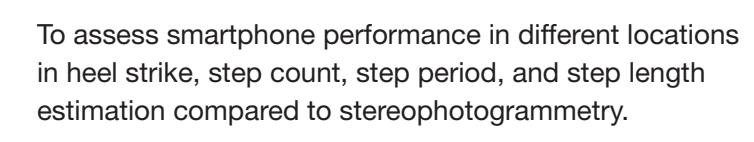

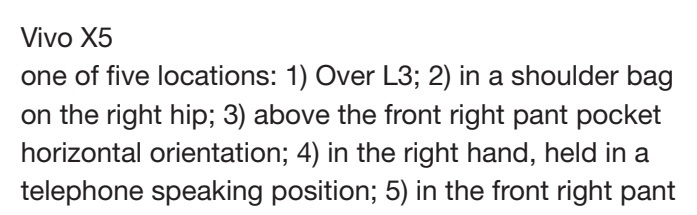

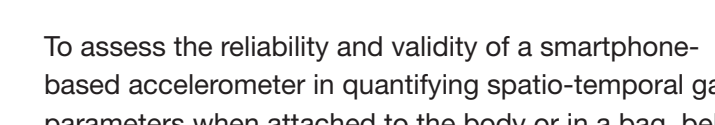

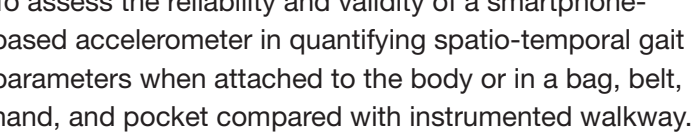

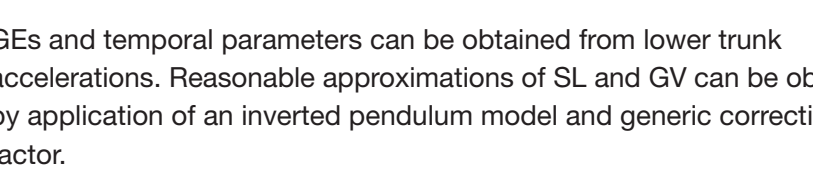

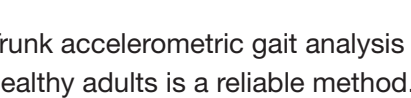

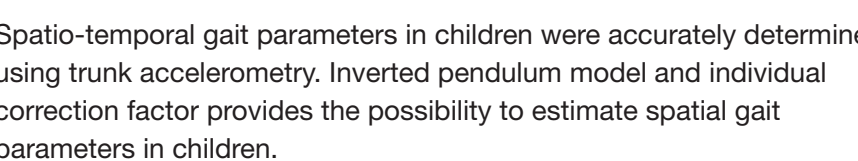

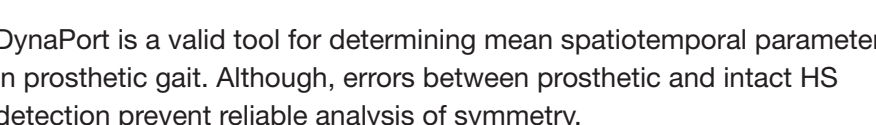

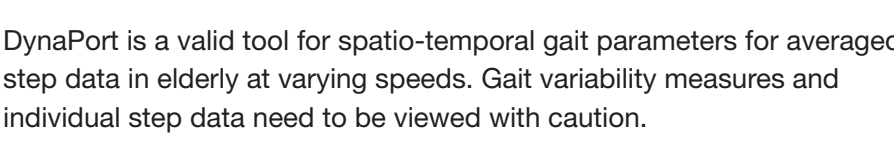

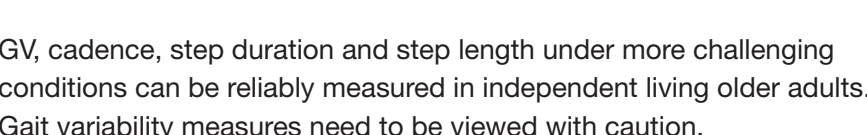

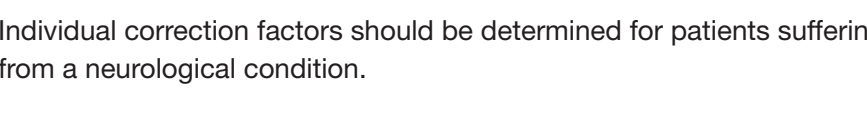

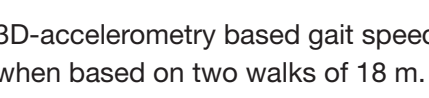

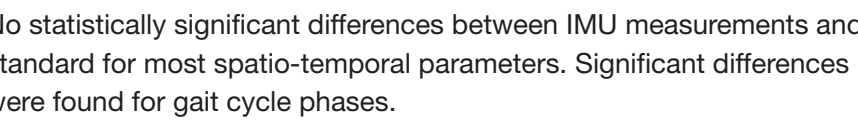

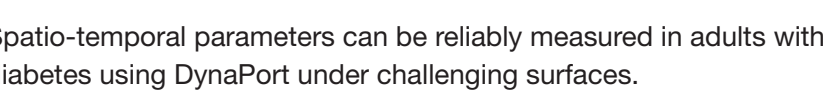

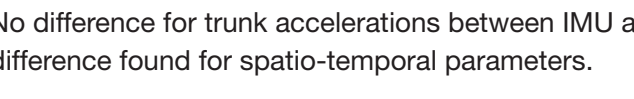

Newly proposed methods led to improved esting
inproved ssimate of individual step lengths.

ascrininatory power between heathry

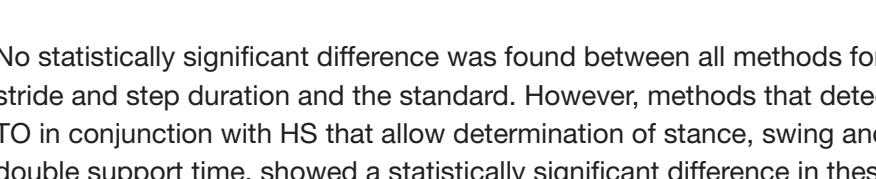

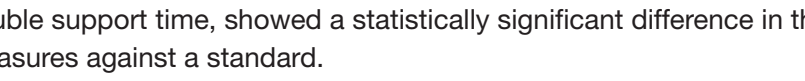

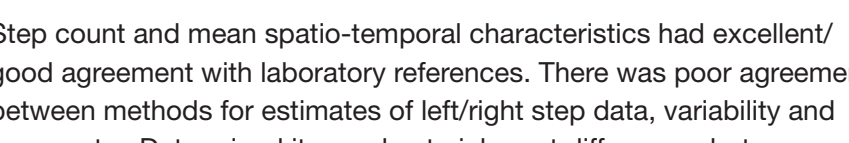

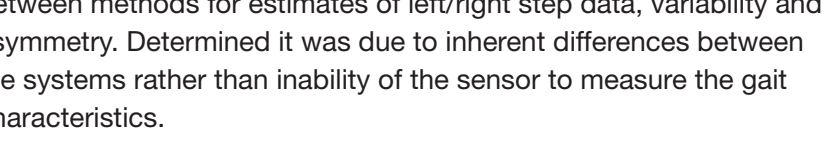
Gait varability a a

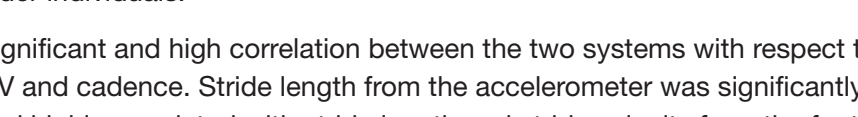
Wessure system.

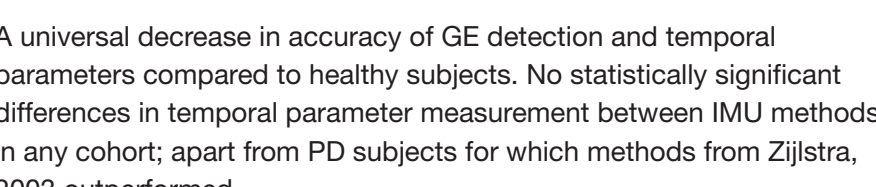

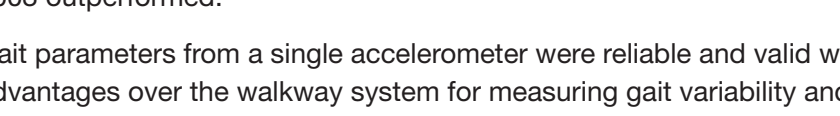

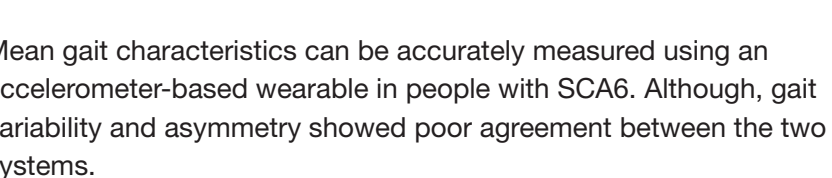

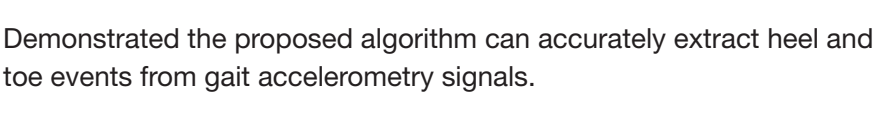

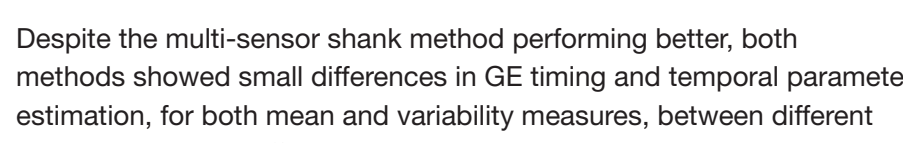

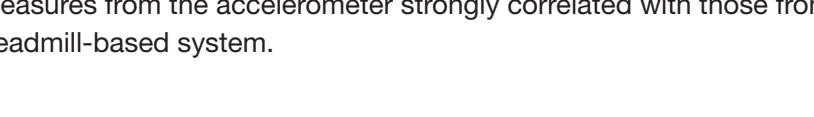

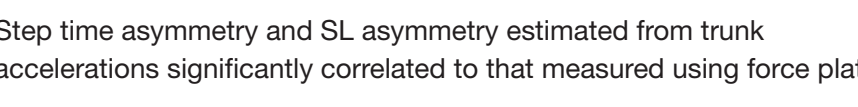

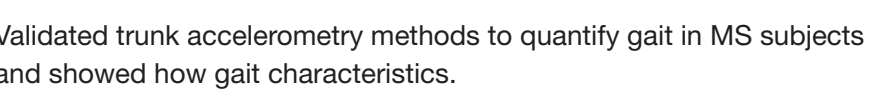

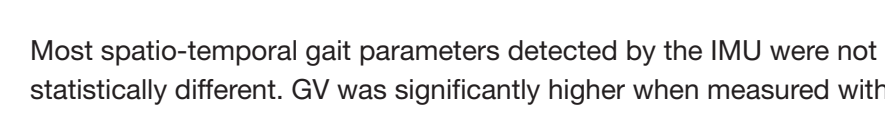

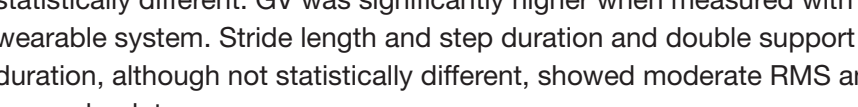

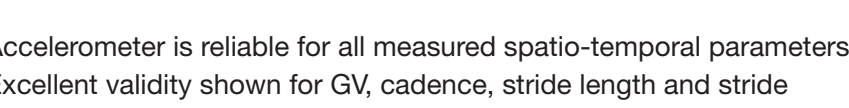

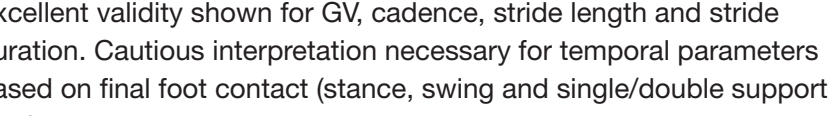

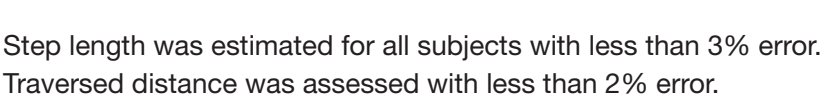

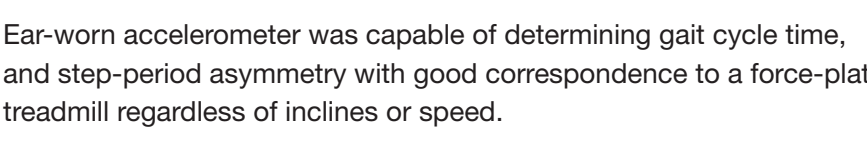

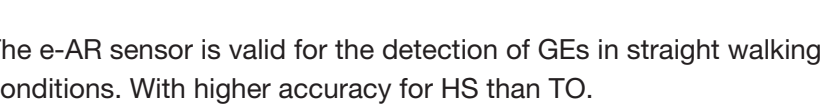

Good agreament between
of GES sand step trevuency.

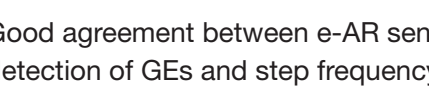

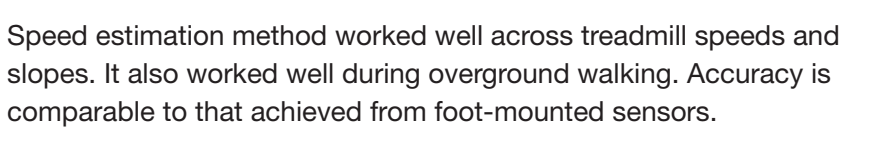

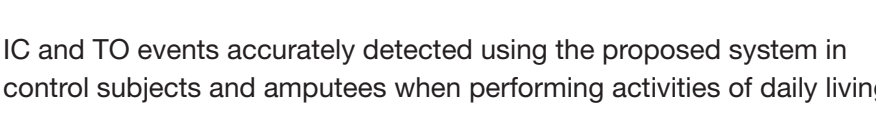

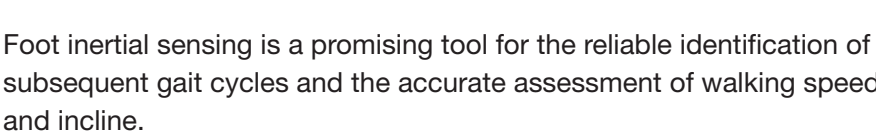

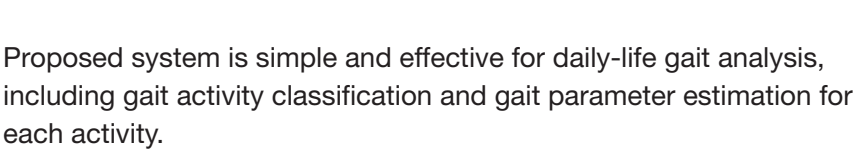

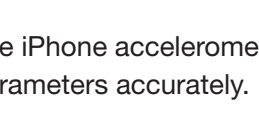

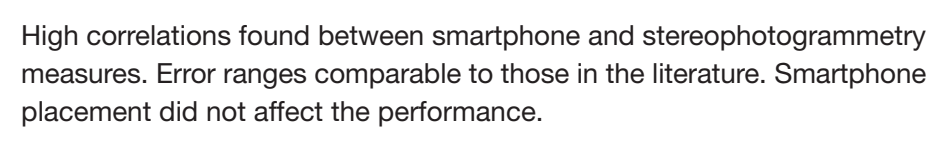

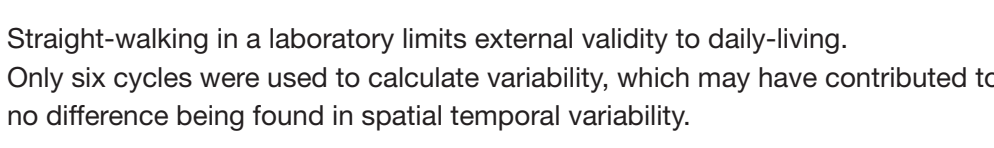

Used sensors of of tifterent mass to the original study design and a barefioc

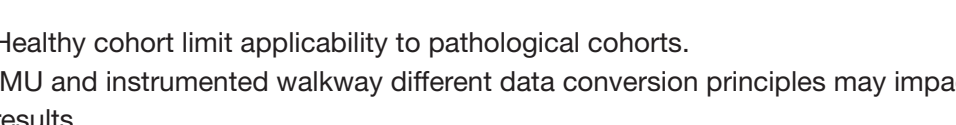

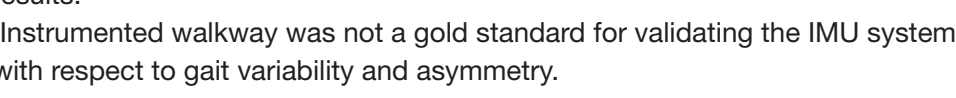

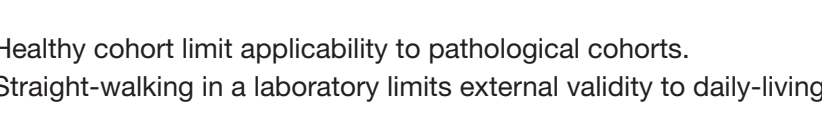

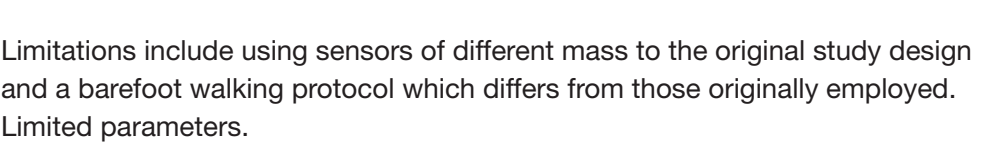

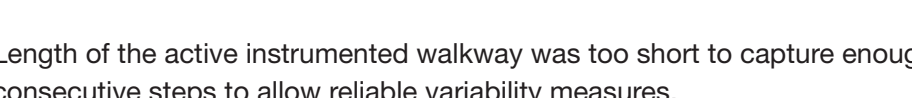
istrumentide walkway was not a gold stander

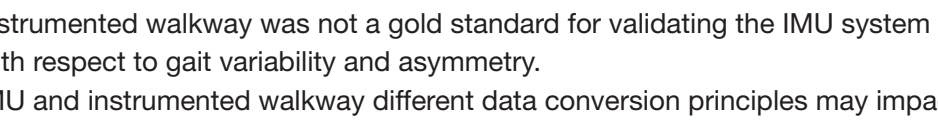

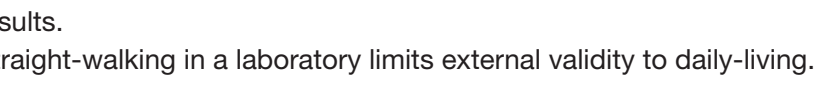

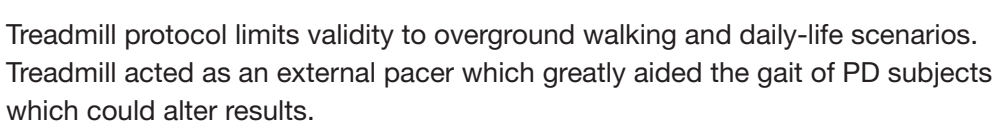
Imall samples size (n=10) of heathy subjects initits appicicability to pathological

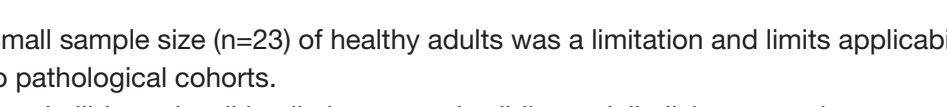
nal validy to doaly-lving scenan as

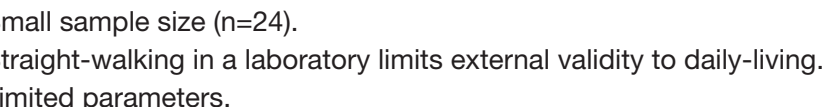

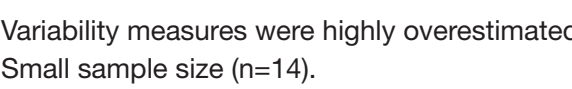

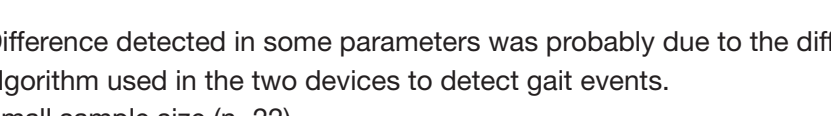

mporal paraneters at a singles sped night have an impect

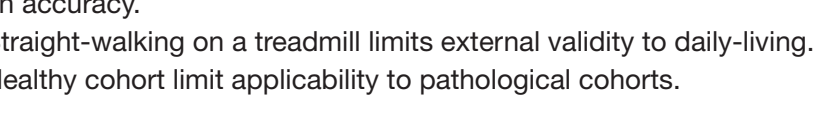

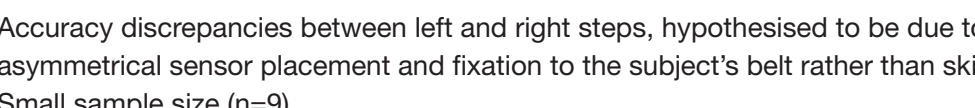
Heathy conot init appicicabily to pathological conots

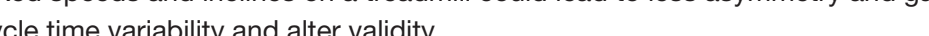

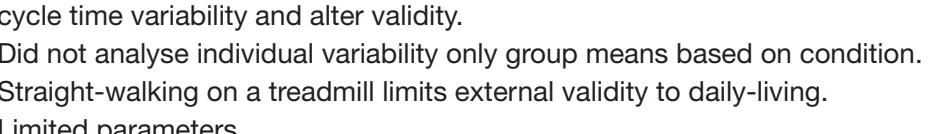

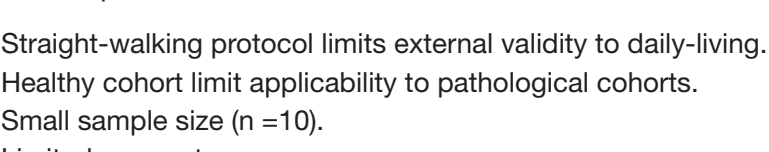

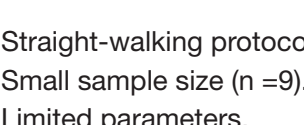

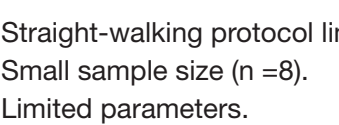

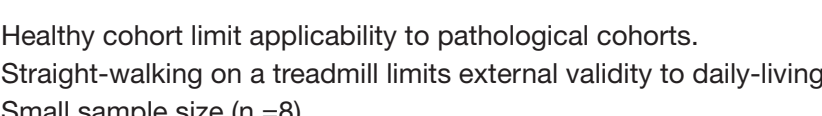

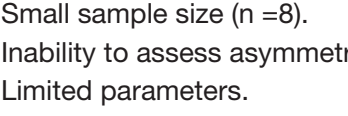

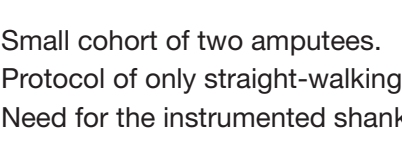

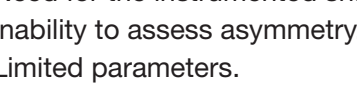

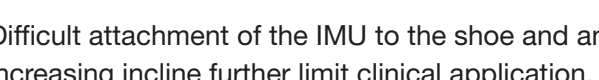

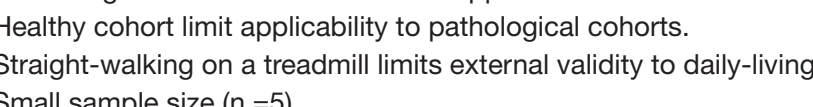

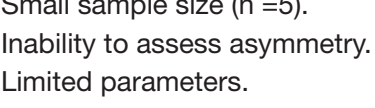

Dificulties were present with
HS
Inability to assess asymmetry
Limited parameters.

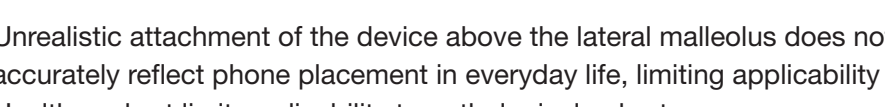

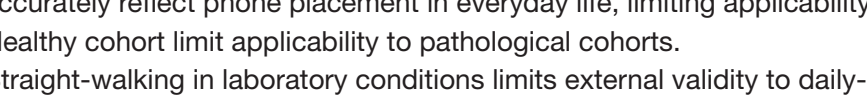

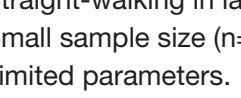

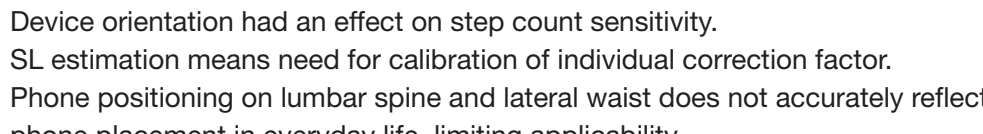

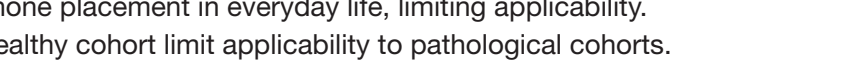

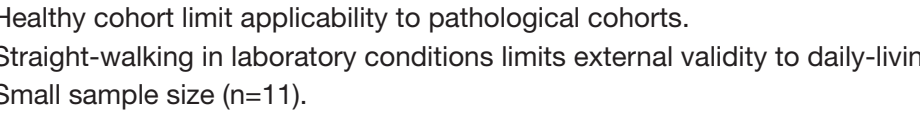
mitations in assessing gatat at sow speeds reduces appication to pathologe

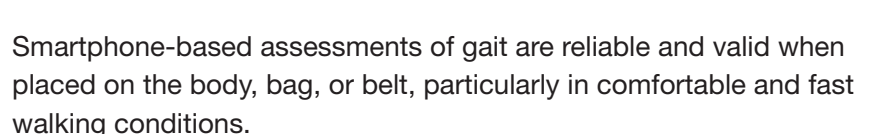


\begin{tabular}{l}
\hline Reference \\
\hline Trunk
\end{tabular}

Auvinet te al, 2003

Moe-Nilssen et al., 2003

Houdijik et al, , 2008

Mizuike et al., 2009

Maquet et al., 2010

Lamoth et al., 2010

Bautmans et al, 2011

Esser et al., 2011

Lamoth et al., 2011

Meijer et al., 2011

Bolink et al., 2012

De Bruin et al., 2012

Hojan et al., 2014

Dalton et al., 2013

Doi et al, 2013

Esser et al., 2013

losa et al., 2013

Manikowska et al,, 2013

Manikowska et al., 2013

Collett et al., 2014

Herman et al, 2014

Pau et al., 2014

Saether et al., 2014

Arvin et al., 2015

Demonceau et al., 2015

Martinez-Ramirez et al., 2015

Matsushima et al., 2015

Perrochon et al, 2015

Rapp et al, 2015

Terrier \& Reynard, 2015

Awotidebe et al., 2016

Barden et al, 2016

Clermont et al., 2016

Del Din et al., 2016

Gillain et al, 2016

Hatanaka et al, 2016

Henderson et al., 2016

Hickey et al., 2016

Martinez-Ramirez et al., 2016

Mutoh et al., 2016

Pau et al., 2016

Van Schooten et al., 2016

Del Din et al., 2017

Pau et al., 2017

Esser et al, 2018

Pau et al., 2018

Storm et al., 2018

Tanigawa et al, 2018

Waist

Terrier et al., 2009

Yang et al., 2011

Terrier et al., 2017

Foot

Chung et al., 2012

Angthong \& Veljkovic, 2018

Ear

Atallah et al, 2014

Jarchi et al, 2016

Smart device

Kosse et al., 2016

Mobbs et al., 2018

Soangra \& Lockhart, 2018

Sensor(s)/Placement

Locometrix (tri-axial accelerometer) over L3-4.

Triaxial, piezoresistant accelerometer (Logger Technologi, Malmö, Sweden) over lower back

DynaPort Minimod (McRoberts BV, The Hague, The Netherlands) consisting of a triaxial laccelerometer.

Tri-axial accelerometer (RF-H48C, Hitachi Metals, Ltd., Japan) over L3.

Locometrix (tri-axial accelerometer) over L3-4.

DynaPort MiniMod (MCRoberts BV, The Hague, The Netherlands) consisting of a triaxial accelerometer
over L3

DynaPort MiniMod (MCRoberts BV, The Hague, The Netherlands) consisting of a triaxial accelerometer
placed over S2.

IMU (MTx, Xsens, The Netherlands) containing a Tri-axial accelerometer, gyroscope and magnetometer,
attached over L4.

DynaPort MiniMod (MCRoberts BV, The Hague, The Netherlands) consisting of a triaxial accelerometer
over L3.

DynaPort MiniMod (McRoberts BV, The Hague, The
Over posterior sacrum at body's centre of mass.

MicroStrain® Inertia-Link@ was used containing gyroscopes and accelerometers. Attached over dorsal side of the pelvis between both posterior superior iliac spines.

DynaPort Minimod (McRoberts BV, The Hague, The Netherlands) consisting of a triaxial accelerometer

DynaPort Minimod (MCRoberts BV, The Hague, The Netherlands) consisting of a triaxial accelerometer

AD_BRC sensor (triaxial accelerometer) attached to upper sternum

Tri-axial accelerometer (MVP-RF8, MicroStone, Nagano, Japan) attached to L3.

IMU (Pi-Node, Philips, Netherlands) over L4.

FreeSense (Sensorize s.r.l., Rome, Italy) containing a tri-axial accelerometer at L2-3.

DynaPort Minimod (McRoberts BV, The Hague, The Netherlands) consisting of a triaxial accelerometer.
Over posterior sacrum at body's centre of mass.

DynaPort Minimod (McRoberts BV, The Hague, The Netherlands) consisting of a triaxial accelerometer.
Over posterior sacrum at body's centre of mass.

IMU device (Pi-node, Philips, Netherlands) containing a tri-axis accelerometer fitted over L4.

DynaPort MiniMod (McRoberts BV, The Hague, The Netherlands) consisting of a triaxial accelerometer
fixed over the lower back.

Tri-axial accelerometer (G-Walk, BTS Bioengineering S.p.A., Italy) attached over L4-L5.

(MTx. XSens, Enschede, NL) attached over L3 contains tri-axial units of accelerometers, gyroscopes, and magnetometers.

DynaPort MiniMod (MCRoberts BV, The Hague, The Netherlands) consisting of a triaxial accelerometer
placed over L5.

IMU (MTX, Xsens, The Netherlands) containing a Tri-axial accelerometer, gyroscope and magnetometer
over the lumbar spine.

Triaxial accelerometer (Jukudai Mate; Kissei Comtec Co., Ltd., Matsumoto, Japan) fixed over $\llcorner 3$.

Locometrix (tri-axial accelerometer) over L3-4.

IMU (Humotion, Münster, Germany) featuring a tri-axis accelerometer and tri-axis gyroscope attached
over L4-5.

Tr-axial accelerometer (Physilog" System, Gaitup, Lausanne, Switzerland) $5 \mathrm{~cm}$ below sternal notch.

IIU Containing tri-axial accelerometer, gyroscope and magnetometer (G-Walk, BTS Bioengineering S.P.A.
Italy) attached over L.

Triaxial accelerometer (GENEActiv, Cambridgeshire, UK) attached over L3.

tri-axial accelerometer (GENEActiv, Cambridge, UK), attached near COM.

Tri-axial accelerometer (Axivity AX3, York, UK) over L5.

Locometrix (tri-axial accelerometer) over L-4-4.

triaxial accelerometer (Mimamori-Gait円 System, LSI Medience Corp., Tokyo, Japan) back of waist. DynaPort Minimod (McRoberts BV, The Hague, The Netherlands) consisting of a triaxial accelerometer
placed on lowert runk. Axivity AX3 sensor (Axivity, York, UK) located on L5.

Tracker MTx (XSENS; Xsens Technologies B.V. Enschede, The Netherlands) featuring tri-axis

Tri-axial accelerometer (MG-M1110-HW, LSI Medience, Tokyo, Japan) over L3.

IMU containing tri-axial accelerometer, gyroscope and magnetometer (G-Walk, BTS Bioengineering S.P.A,
Italy) attached over L4-5.

DynaPort Minimod (MCRoberts BV, The Hague, The Netherlands) consisting of a triaxial accelerometer
over L5.

Tri-axial accelerometer (Axivity AX3, York, UK) on the lower back

Triaxial accelerometer fixed to participant's sacrum.

IMU (tri-axial accelerometer and gyroscope) fixed to the lower back.

Tri-axial accelerometer (G-Walk, BTS Bioengineering S.P.A., Italy) attached over L4-5.

Tri-axial accelerometer (MoveMonitor, Version 2.8.1, Mc Roberts, The Hague, The Netherlands) positioned
at the lower back. The other two IMUs positioned at each ankle, just above the malleoli.

MVP-RF-8, MicroStone Co., Nagano, Japan) that contained a triaxial angular rate gyroscope and a

Physilog system (BioAGM, Switzerland), triaxial accelerometer lateral waist.

Tri-axial accelerometer at lateral waist.

Actigraph wGT3X-BT activity monitor (Actigraph, Pensacola, FL, USA) lateral waist.

Triaxial accelerometer on superior aspect of right foot.

Foot pod (Garmin Ltd., Kansas City, USA) strapped to dorsum of foot.

e-AR sensor (containing a tri-axial MEMS accelerometer) fitted behind the ear.

e-AR sensor (containing a tri-axial MEMS accelerometer) fitted behind the ear.

iPod touch G4 (iOS 6, Apple Inc.) fixed over L3.

Apple Watch (Apple, San Francisco, CA, USA) on the wrist.

Apple iPhone 5 (iPhone 5, Apple Inc., Cupertino, CA, USA) on lateral waist.

Application

Gait abnormalities in elderly fallers versus healthy controls

Discriminate between children with dyslexia and healthy controls based on gall

Assessment of spatiotemporal parameters of amputee gait.

Describe gait of stroke patients delineate from healthy controls

Delineation of elderly, Alzheimer's and mild cognitive impairment subjects by gait analysis under single
and dual task protocols.

Differentiate transfemoral amputees and healthy controls based on gal

Delineating healthy elderly and young subjects from elderly fallers from gait.

$\mathrm{PD}$, muscular dystrophy, MND, stroke survivors and healthy subjects.

Effect of dual task conditions on gait in Dementia subjects and healthy controls.

Gait in post-stroke patients versus healthy controls.

Objective assessment of total knee replacement in osteoarthritis patients and differentiating from healthy
controls.

Determining severity of peripheral neuropathy in diabetics.

External breast prosthesis effect on gait after mastectom

Differentiating between pre-manifest $\mathrm{HD}$, manifest $\mathrm{HD}$ and healthy controls.

Effect of multicomponent exercise on gait in elderly with MCI.

Differentiate PD patients and healthy controls

Differentiating Cerebral Palsy children from healthy controls during running and walking

Gait patterns in pre and post-menopausal women.

Effect of hippotherapy on gait in cerebral palsy children.

Delineating HD patients from healthy controls and determining disease severity using gait

Quantify motor differences in PD subtypes and propose a classification scheme.

Effect of physical activity on balance and gait of older adults.

Gait characteristics in cerebral palsy versus healthy children.

Effect of unilateral hip abductor muscle fatigue on gait in older adults.

Delineating PD patients from healthy controls and determining disease severity.

Frailty assessment based on gait assessment.

Delineating Ataxia patients from healthy controls and determining disease duration.

Effects of exercise on gait in dementia patients.

Efficacy of rehabilitation for patients after hip arthroplasty.

Effect of aging on gait parameters.

Delineating between type 2 diabetes patients and healthy controls

Compare regularity and symmetry of gait between knee osteoarthritis (OA) and healthy controls Delineating between knee osteoarthritis patients and age-matched controls.

Delineating between PD patients and age-matched controls.

Determining risk of developing $\mathrm{AD}$ from $\mathrm{MCl}$ based on gait.

Gait comparison in Progressive Supranuclear Palsy and PD and delineating from heathy controls.

Effect of rivastigmine on gait variability in $\mathrm{PD}$.

Differentiating patients with spinocerebellar ataxia type-6 from healthy controls.

Dual task walking performance in frail populations with and without MCl against controls.

Effects of hippotherapy on gait and balance ability of children and adolescents with cerebral palsy.

Gait measures in MS and correlation with patient-reported outcomes. Determining MS severity.

Gait characteristics as a predictor of falls in older subjects

Gait characteristics in fallers and non-fallers with and without PD.

Gait in early MS subjects compared to healthy control.

Gait analysis to detect peripheral neuropathy in diabetes patients.

Effect of texting while walking on gait in MS patients compared to healthy controls

Characterise gait in MS patients in laboratory and free-living conditions and determine severity.

Relationship of lumbopelvic pain with gait in pregnant patients.

Assess the effect of prescription footwear on gait quality in patients after ankle/foot fractures.

Differentiating PD and healthy controls.

Delineating patients with chronic pain of lower limbs and healthy controls from free-living gait.

Delineating patients with Alzheimer's disease (AD) from heathy controls.

Relationship of patient-reported outcomes and quality-of-life with gait characteristics in patients with foot-

Post-operative recovery of orthopaedic patient.

To assess recovery in anterior cruciate ligament injury patients after surgery.

Assess gait variability changes related to aging.

Gait analysis for objective recovery measures following lumbar microdiscectomy.

Identifying CVD patients likely to have post-operative adverse outcomes based on gait analysis.
Parameters Measured

GV and stride frequency, length, symmetry and regularity

GV, cadence and SL.

Step count, SL, step duration and GV.

GV SL, stride duration, cadence, stride regularity and RMS.

GV, stride frequency, stride length, stride symmetry and stride regularity.

GV, stride time, stride time variability, non-linear measures (variability, regularity, stability) and RMS

GV, step time, step time symmetry, step regularity and stride regularity

Step time, SL and GV.

stability.

$\mathrm{GV}$, cadence, gait symmetry and bilateral coordination of gait.

GV, cadence, SL, step time, step time variability and step time asymmetry.

SL, GV, step duration and cadence.

GV, SL, step time, cadence and step time asymmetry

SL, stride length, cadence, step time, GV, Step time/SLstride length variability, step asymmetry and step
stride regularity

GV, stride time, stride length and $\mathrm{HF}$.

Cadence, stride length, GV, cadence variability, stride length variability and non-linear measures (gail
variability).

GV, SL, step duration, RMS and HR.

GVV, SL, cadence, stance phase duration, swing phase duration, single support duration, double support

GV, cadence, SL, stride length and left-right symmetry.

GV, step time, step time variability, cadence, stride length, stride length variability and non-linear measures
(gait variability and symmetry). Step count, GV, stride time variability, stride regularity and cadence.

$\mathrm{SL}, \mathrm{GV}$, gait cycle duration, stance duration, swing duration and double support duration

GV, cadence, SL, step time, stride regularity and symmetry.

Stride time, stride time variability, step symmetry, stance time, HR and non-linear measures (gait stability.

Cadence, SL, gait regularity and symmetry.

GV, cadence, step regularity, stride regularity, gait symmetry, step time, step time variability, RMS and HR.

GV, cadence, SL, gait regularity and RMS.

GV, stride frequency, stride length, gait symmetry and gait regularity

GV and gait symmetry.

GV, non-linear measures (gait stability). RMS and walk-ratio.

GV, SL, stride length and cadence.

Stride time, step time, stride regularity, step regularty and gait symmetry

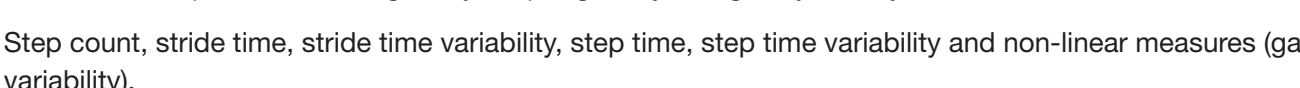

GV, SL, swing time variability, GV variability, SL variability, stpe time variability, stance time variability, step time, swing

Stride frequency, SL, gait symmetry and regularity.

GV, SL, cadence, step time, step time variability and double support time.

GV and step time variability.

GV, SL, swing time variability, step time, swing time, stance time, step time variability, SL variability, stance time variab

GV, step and stride regularity, gait symmetry, step time variability, RMS, gait stability and HR.

Cadence, SL and GV.

Stride length, GV, cadence, stance duration, swing duration and double support duration.

Stride length, GV, stride time variability, GV variability, stride length variability, stride length, gait symmetty,
gait variability, non-linear measures (gait smoothness, complexity, intensity) and stride frequency. Step count, mean bout length, step time, swing time, stance time, step time asymmetry, swing time
asymmetry stance time asymmetry, SL asymmetry, step velocity, SL, swing time variability, step time
variability, stance time variability, GV and SL variability.

Cadence, speed, stride length, stance, swing and double support phase duration.

Step time, cadence, stride length, GV and non-linear measures (gait control variables).

Stride length, GV, cadence, stance duration, swing duration and double support duration

HS, TO, stride time, step time, stance time, swing time, stride time variability, step time variability, stance
time variability, swing time variability, step count, GV

Gait symmetry and stride variability.

Stride regularity and stride symmetry.

Cadence, step regularity, step symmetry and stride regularity

Cadence, stride regularity and non-linear measures (intensity, dynamic stability).

Stride length, stride frequency, GV, cadence, stance phase duration and stance phase variability

Distance travelled, step count, SL, cadence and GV.

Stride duration and step time asymmetry.

Gait asymmetry.

Stride time, stride time variability, GV, RMS and non-linear measures (gait stability).

GV and non-linear measures (gait variability). 
Table S3 Risk of bias assessment of validity and reliability studies

\begin{tabular}{|c|c|c|c|c|c|c|c|c|c|c|c|c|c|c|}
\hline \multirow{2}{*}{ Article (author, year) } & \multicolumn{4}{|c|}{ Patient Selection } & \multicolumn{3}{|c|}{ Index Test } & \multicolumn{3}{|c|}{ Reference Standard } & \multicolumn{3}{|c|}{ Flow and Timing } & \multirow{2}{*}{$\frac{\text { Overall }}{5.1}$} \\
\hline & 1.1 & 1.2 & 1.3 & 1.4 & 2.1 & 2.2 & 2.3 & 3.1 & 3.2 & 3.3 & 4.1 & 4.2 & 4.3 & \\
\hline Atallah et al., 2014 & CS & Y & Y & Y & Y & NA & $\mathrm{Y}$ & Y & Y & Y & Y & $\mathrm{Y}$ & Y & ++ \\
\hline Bautmans et al., 2011 & CS & $\mathrm{Y}$ & Y & Y & NA & NA & Y & NA & NA & NA & NA & NA & Y & ++ \\
\hline Brandes et al., 2006 & CS & Y & Y & Y & Y & NA & Y & CS & Y & CS & $\mathrm{Y}$ & $\mathrm{Y}$ & Y & + \\
\hline Bugane et al., 2012 & CS & Y & Y & Y & Y & NA & Y & Y & $Y$ & Y & Y & Y & Y & ++ \\
\hline Byun et al., 2016 & CS & Y & Y & Y & Y & NA & Y & Y & Y & $Y$ & Y & $Y$ & CS & ++ \\
\hline Dalton et al., 2013 & CS & Y & Y & Y & Y & NA & Y & Y & Y & Y & Y & Y & Y & ++ \\
\hline De Bruin et al., 2012 & $\mathrm{~N}$ & Y & Y & CS & NA & NA & Y & NA & NA & NA & NA & NA & $\mathrm{N}$ & + \\
\hline De Ridder et al., 2019 & CS & Y & Y & $\mathrm{Y}$ & $\mathrm{Y}$ & NA & Y & Y & $Y$ & $\mathrm{Y}$ & Y & $\mathrm{Y}$ & CS & ++ \\
\hline Esser et al., 2012 & CS & Y & Y & $\mathrm{Y}$ & Y & NA & Y & Y & Y & Y & Y & $\mathrm{Y}$ & CS & ++ \\
\hline Godfrey et al, 2014 & CS & Y & Y & Y & Y & NA & Y & Y & Y & $\mathrm{Y}$ & Y & $\mathrm{Y}$ & CS & ++ \\
\hline Godfrey et al, 2015 & CS & Y & Y & CS & Y & NA & Y & Y & Y & $Y$ & $\mathrm{Y}$ & Y & N & + \\
\hline Grimpampi et al., 2015 & CS & Y & Y & Y & NA & NA & Y & NA & NA & NA & NA & NA & Y & ++ \\
\hline Hartmann et al., 2009 & CS & Y & Y & Y & Y & NA & Y & Y & Y & Y & Y & $\mathrm{Y}$ & Y & ++ \\
\hline $\begin{array}{l}\text { Hartmann et al., } 2009 \\
\text { (different surfaces) }\end{array}$ & CS & Y & Y & $\mathrm{Y}$ & NA & NA & Y & NA & NA & NA & NA & NA & $\mathrm{N}$ & + \\
\hline Henriksen et al., 2004 & CS & Y & Y & $\mathrm{Y}$ & NA & NA & Y & NA & NA & NA & NA & NA & Y & ++ \\
\hline Hickey et al., 2016 & CS & Y & Y & CS & Y & NA & Y & $\mathrm{Y}$ & Y & Y & Y & Y & Y & ++ \\
\hline Houdijk et al., 2008 & CS & Y & Y & $\mathrm{Y}$ & $\mathrm{Y}$ & NA & Y & Y & Y & $\mathrm{Y}$ & Y & $Y$ & Y & ++ \\
\hline Jarchi et al., 2014 & CS & Y & Y & CS & Y & NA & Y & $\mathrm{Y}$ & Y & $\mathrm{Y}$ & Y & Y & $\mathrm{Y}$ & ++ \\
\hline Jarchi et al., 2015 & cs & Y & Y & Y & Y & NA & Y & Y & Y & Y & Y & Y & N & + \\
\hline Jarchi et al., 2016 & CS & Y & Y & CS & $\mathrm{Y}$ & NA & Y & Y & Y & $\mathrm{Y}$ & $Y$ & $\mathrm{Y}$ & Y & ++ \\
\hline Köse et al., 2012 & CS & Y & Y & CS & Y & NA & Y & Y & Y & Y & Y & Y & Y & ++ \\
\hline LeMoyne et al., 2010 & $\mathrm{~N}$ & Y & Y & CS & NA & NA & Y & NA & NA & NA & NA & NA & Y & + \\
\hline Li et al., 2010 & CS & Y & Y & Y & Y & NA & Y & $\mathrm{Y}$ & Y & $\mathrm{Y}$ & Y & $\mathrm{Y}$ & Y & ++ \\
\hline Lim \& Lee, 2017 & CS & Y & Y & Y & Y & NA & Y & Y & Y & Y & Y & Y & CS & ++ \\
\hline Maqbool et al., 2017 & CS & Y & Y & CS & Y & NA & Y & Y & Y & $\mathrm{Y}$ & $Y$ & $Y$ & Y & ++ \\
\hline McCamley et al., 2012 & CS & Y & Y & Y & Y & NA & Y & $\mathrm{Y}$ & Y & Y & Y & $Y$ & CS & ++ \\
\hline Oyake et al., 2017 & CS & Y & Y & $\mathrm{Y}$ & Y & NA & Y & Y & Y & $\mathrm{Y}$ & $Y$ & $\mathrm{Y}$ & CS & ++ \\
\hline Park \& Woo, 2015 & CS & Y & Y & CS & Y & NA & Y & Y & Y & Y & Y & $\mathrm{Y}$ & CS & + \\
\hline Pepa et al., 2017 & CS & Y & Y & CS & Y & NA & Y & Y & Y & Y & Y & Y & CS & + \\
\hline Sabatini et al., 2005 & CS & Y & Y & CS & $\mathrm{Y}$ & NA & Y & Y & Y & $\mathrm{Y}$ & $\mathrm{Y}$ & $\mathrm{Y}$ & Y & ++ \\
\hline Sejdic et al., 2016 & CS & Y & Y & Y & Y & NA & Y & Y & $Y$ & Y & Y & $Y$ & CS & ++ \\
\hline Silsupadol et al., 2017 & CS & Y & Y & CS & $\mathrm{Y}$ & NA & Y & Y & $Y$ & $Y$ & $Y$ & $Y$ & Y & ++ \\
\hline Song \& Kim, 2018 & CS & Y & Y & Y & Y & NA & Y & Y & Y & Y & Y & $Y$ & CS & ++ \\
\hline Storm et al., 2016 & CS & Y & Y & $\mathrm{Y}$ & $\mathrm{Y}$ & NA & Y & Y & $Y$ & $Y$ & $\mathrm{Y}$ & $Y$ & Y & ++ \\
\hline Storm et al., 2018 & CS & Y & Y & Y & Y & NA & Y & Y & Y & $\mathrm{Y}$ & $\mathrm{Y}$ & $\mathrm{Y}$ & $\mathrm{N}$ & + \\
\hline Trojaniello et al., 2014 & CS & Y & $\mathrm{Y}$ & Y & Y & NA & Y & Y & $Y$ & $Y$ & Y & Y & Y & ++ \\
\hline Trojaniello et al., 2015 & CS & Y & Y & Y & $\mathrm{Y}$ & NA & Y & Y & $Y$ & $\mathrm{Y}$ & $Y$ & $Y$ & CS & ++ \\
\hline Zago et al., 2018 & CS & Y & Y & Y & Y & NA & Y & Y & Y & Y & Y & $Y$ & CS & ++ \\
\hline Zijlstra \& Hof, 2003 & CS & Y & Y & Y & $\mathrm{Y}$ & NA & Y & Y & $\mathrm{Y}$ & $\mathrm{Y}$ & Y & $Y$ & Y & ++ \\
\hline Zijlstra \& Zijlstra, 2013 & CS & $\mathrm{Y}$ & Y & Y & Y & NA & Y & Y & CS & $Y$ & $Y$ & $Y$ & $\mathrm{Y}$ & ++ \\
\hline Collett et al., 2014* & & & & & & & sesse & using & & & & & & \\
\hline Esser et al., 2011* & & & & & & & sesse & using & & & & & & \\
\hline
\end{tabular}

$\mathrm{Y}$, yes; $\mathrm{N}, \mathrm{no}$; NA, not applicable; CS, can’t say; "+" denotes acceptable quality in minimizing bias; "++" denotes high quality in minimizing bias; NOS, Newcastle-Ottawa Scale. *, refer Appendix 1. 
Appendix 1 Risk of bias assessment of clinical applicability studies

\begin{tabular}{|c|c|c|c|c|}
\hline Study (Author, Year) & Selection & Comparability & Exposure & Results \\
\hline Angthong, 2018 & 2 & 2 & 2 & Fair \\
\hline Atallah, 2014 & 2 & 1 & 2 & Fair \\
\hline Auvinet, 2003 & 3 & 0 & 2 & Poor \\
\hline Awotidebe, 2016 & 4 & 1 & 2 & Good \\
\hline Barden, 2016 & 3 & 2 & 2 & Good \\
\hline Bautmans, 2011 & 4 & 2 & 2 & Good \\
\hline Bolink, 2012 & 3 & 1 & 2 & Good \\
\hline Chung, 2012 & 1 & 1 & 2 & Poor \\
\hline Clermont, 2016 & 3 & 1 & 2 & Good \\
\hline Collett, 2014 & 3 & 2 & 2 & Good \\
\hline Dalton, 2013 & 3 & 2 & 2 & Good \\
\hline De Bruin, 2012 & 2 & 2 & 2 & Fair \\
\hline Del Din, 2016 & 3 & 1 & 2 & Good \\
\hline Del Din, 2017 & 3 & 1 & 2 & Good \\
\hline Demonceau, 2015 & 3 & 2 & 2 & Good \\
\hline Esser, 2011 & 1 & 0 & 1 & Poor \\
\hline Esser, 2013 & 4 & 2 & 2 & Good \\
\hline Esser, 2018 & 3 & 1 & 2 & Good \\
\hline Gillain, 2016 & 2 & 2 & 2 & Fair \\
\hline Hatanaka, 2016 & 3 & 1 & 2 & Good \\
\hline Herman, 2014 & 3 & 2 & 2 & Good \\
\hline Hickey, 2016 & 4 & 1 & 2 & Good \\
\hline Hojan, 2014 & 3 & 1 & 2 & Good \\
\hline losa, 2013 & 2 & 2 & 2 & Fair \\
\hline Jarchi, 2016 & 2 & 1 & 1 & Poor \\
\hline Kosse, 2016 & 3 & 1 & 2 & Good \\
\hline Lamoth 2011 & 3 & 1 & 2 & Good \\
\hline Lamoth, 2010 & 3 & 1 & 1 & Poor \\
\hline Manikowska, 2013 (postmenopausal women) & 3 & 1 & 2 & Good \\
\hline Maquet, 2010 & 3 & 2 & 2 & Good \\
\hline Martinez-Ramirez, 2015 & 3 & 1 & 1 & Poor \\
\hline Martinez-Ramirez, 2016 & 3 & 1 & 2 & Good \\
\hline Matsushima, 2015 & 2 & 1 & 2 & Fair \\
\hline Meijer, 2011 & 3 & 2 & 2 & Good \\
\hline Mizuike, 2009 & 4 & 1 & 2 & Good \\
\hline Moe-Nilssen, 2003 & 4 & 2 & 3 & Good \\
\hline Pau, 2016 & 4 & 0 & 2 & Poor \\
\hline Pau, 2017 & 4 & 0 & 2 & Poor \\
\hline Pau, 2018 & 3 & 1 & 2 & Good \\
\hline Perrochon, 2015 & 2 & 2 & 2 & Fair \\
\hline
\end{tabular}




\begin{tabular}{|l|lllc|}
\hline Rapp, 2015 & 3 & 2 & 1 & Poor \\
\hline Saether, 2014 & 4 & 2 & 2 & Good \\
\hline Soangra, 2018 & 2 & 1 & 2 & Fair \\
\hline Storm, 2018 & 3 & 1 & 2 & Good \\
\hline Tanigawa, 2018 & 3 & 0 & 2 & Poor \\
\hline Terrier, 2009 & 1 & 2 & 2 & Poor \\
\hline Terrier, 2015 & 3 & 1 & 2 & Good \\
\hline Terrier, 2017 & 3 & 1 & 2 & Good \\
\hline Van Schooten, 2016 & 3 & 2 & 2 & Good \\
\hline Yang, 2011 & 3 & 0 & 2 & Poor \\
\hline
\end{tabular}

\begin{tabular}{|c|c|c|c|c|c|c|c|c|c|c|c|c|}
\hline \multicolumn{13}{|c|}{ Randomised control trials (RCT) } \\
\hline \multirow{3}{*}{$\begin{array}{l}\text { Study (Author, } \\
\text { Year) }\end{array}$} & & \multicolumn{10}{|c|}{ Internal validity } & \multirow{3}{*}{$\begin{array}{c}\text { Overall } \\
\text { assessment }\end{array}$} \\
\hline & \multirow[t]{2}{*}{1.1} & \multirow[t]{2}{*}{1.2} & \multirow[t]{2}{*}{1.3} & \multirow[t]{2}{*}{1.4} & \multirow[t]{2}{*}{1.5} & \multirow{2}{*}{1.6} & \multirow[t]{2}{*}{1.7} & \multicolumn{2}{|c|}{1.8} & \multirow[t]{2}{*}{1.9} & \multirow[t]{2}{*}{1.10} & \\
\hline & & & & & & & & $I(\%)$ & $C(\%)$ & & & \\
\hline Doi, 2013* & $Y$ & CS & CS & CS & $\mathrm{Y}$ & $\mathrm{Y}$ & $\mathrm{Y}$ & 4 & 8 & $\mathrm{Y}$ & NA & + \\
\hline Henderson, 2016* & $Y$ & $\mathrm{Y}$ & $\mathrm{Y}$ & $\mathrm{Y}$ & CS & CS & $\mathrm{Y}$ & 15 & 11 & $\mathrm{Y}$ & NA & ++ \\
\hline Pau, 2014* & $Y$ & $\mathrm{Y}$ & CS & CS & CS & $\mathrm{Y}$ & $\mathrm{Y}$ & 0 & 0 & $\mathrm{Y}$ & NA & + \\
\hline
\end{tabular}

\begin{tabular}{|l|l|}
\hline \multicolumn{2}{|c|}{ Not assessed } \\
\hline Houdijk, 2008 & Assessed using SIGN \\
\hline Arvin, 2015 & Self-controlled before-after study \\
\hline Manikowska, 2013 & Case series \\
\hline Mobbs, 2018 & Case report \\
\hline Mutoh, 2016 & Case series \\
\hline
\end{tabular}

NOS interpretation:

Good quality: $\geq 3$ stars in selection domain AND $\geq 1$ star in comparability domain AND $\geq 2$ stars in outcome/exposure domain.

Fair quality: 2 stars in selection domain AND $\geq 1$ star in comparability domain AND $\geq 2$ stars in outcome/exposure domain.

Poor quality: $\leq 1$ star in selection domain OR 0 stars in comparability domain $\mathrm{OR} \leq 1$ star in outcome/exposure domain.

$\mathrm{Y}$, yes; N, no; NA, not applicable; CS, can't say; “+" denotes acceptable quality in minimizing bias; "++" denotes high quality in minimizing bias; I (\%), percentage dropout in intervention group; C (\%), percentage dropout in control group. ${ }^{*}$, RCTs were assessed using SIGN checklist for RCTs. 